\title{
The creeping motion of immiscible drops through a converging/diverging tube
}

\author{
By W. L. OLBRICHT† AND L. G. LEAL \\ Department of Chemical Engineering, California Institute of Technology, \\ Pasadena, California 91125
}

(Received 11 August 1980 and in revised form 10 May 1983)

Results of experiments on the low-Reynolds-number flow of liquid drops through a horizontal circular tube with a diameter that varies sinusoidally with axial position are reported. Measurements of the contribution of the drop to the local pressure gradient and the relative velocity of the drop are correlated with the time-dependent drop shape. Both Newtonian and viscoelastic suspending fluids are considered. The viscosity ratio, volumetric flow rate and drop size are varied in the experiment, and both neutrally buoyant and non-neutrally buoyant drops are studied. Comparison with previous results for a straight-wall tube shows that the influence of the tube boundary geometry on the drop shape is substantial, but the qualitative effect of the tube shape depends strongly on the relative importance of viseous forces compared to interfacial tension for the particular experiment. For Newtonian fluids, two modes of drop breakup, which are distinguished by the magnitude of the viscosity ratio, are observed. When the suspending fluid is viscoelastic, both shear-thinning and time-dependent rheological effects are present.

\section{Introduction}

We report in this paper the results of experiments on the creeping flow of immiscible drops through a horizontal circular tube with periodically varying diameter. We have conducted experiments for both Newtonian and viscoelastic suspending fluids. This is part of a general study aimed at understanding the dynamics of two-phase flow through porous media at the scale of the individual channels.

One specific application involves recovery of oil by chemical flooding methods. When the saturation of the dispersed (oil) phase is below a critical value, unrecovered oil resides in the porous matrix in the form of isolated globules. These globules can be displaced by a second fluid provided that viscous forces are made sufficiently large compared to interfacial tension forces which tend to hold the globules fixed in certain equilibrium configurations. The fate of an individual mobilized globule is uncertain, but possibilities include stranding, coalescence with another globule, and breakup into smaller globules (cf. Payatakes 1982); our model study focuses on the detailed dynamics of droplet motion, as well as on possible dispersion processes that can influence the overall recovery of the dispersed phase. It is important to note that many different procedures to accomplish the displacement have been proposed, but most of them involve, at some stage, the use of polymer solutions as displacing fluids and/or to help maintain the integrity of a bank of a separate, interface-active fluid. These fluids typically are non-Newtonian and, therefore, part of our study is aimed

† Present address: School of Chemical Engineering, Cornell University, Ithaca, New York 14853. 
at exposing the effects of suspending phase rheology on the dynamics of droplet motion.

The specific problem we study is the motion of immiscible Newtonian drops through a horizontal circular tube with a diameter that varies sinusoidally with axial position. The 'wavy-wall' configuration is chosen because the geometry produces certain features of the flow kinematics which are expected to be essential to a model of flow in an individual pore, especially for multiphase systems. First, continuity requires that the velocity varies in the streamwise direction, and this means that the flow field is partially extensional. The flow along the centreline of the tube resembles uniaxial elongation in the convergent region and biaxial elongation in the divergent region. The flow kinematics are, however, known only along the centreline of the wavy-wall tube. Fluid elements not coincident with the tube centreline experience an additional rotational component, although the magnitude of the straining part of the velocity-gradient tensor always exceeds the vorticity, provided there is no flow separation. The relative importance of the elongational component of the flow at any given point in the tube increases with the amplitude of variation in the tube diameter. Secondly, the wavy-wall shape means that the flow field is Lagrangian unsteady over a convective timescale which is related to the average velocity and to the 'wavelength' of the tube. This implies that the shape of a drop suspended in the flow for our experiment is time-dependent. Furthermore, even with no drops present, the unsteadiness of the flow can also affect the material behaviour of the viscoelastic suspending fluids.

The creeping motion of immiscible drops in tube flow has been the focus of two previous experimental investigations from this laboratory. Ho \& Leal (1975) considered the motion of neutrally buoyant drops suspended in Stokes flow through a straight-wall circular horizontal tube. Recently, Olbricht \& Leal (1982) examined the effects of a density difference between the drop fluid and the suspending fluid. These papers will be referred to here as (I) and (II) respectively. For the present experiment, we are concerned primarily with the effects of the wavy-wall tube geometry on drop deformation and possible breakup under a wide range of conditions, including both neutrally buoyant and non-neutrally buoyant cases.

In the present study, only a single wavy-wall tube has been used (for details see $\S 2$ ). Thus a comprehensive investigation of tube geometry has not been undertaken, though we recognize that the structure of the interstitial region of real porous media might encompass a wide range in the amplitude and 'period' of streamwise variations in a cross-sectional area. Nevertheless, we ultimately seek to determine also conditions under which the details of drop deformation can affect the overall or macroscale dynamics of multiphase flow through real porous structures. Therefore we have measured certain dynamic quantities, including the contribution of the drop to the local pressure gradient and the velocity of the drop relative to the average two-phase velocity, and we attempt to correlate these measurements with the time-dependent drop shape. In the context of the macroscale problem, the contribution of the drop to the pressure gradient is a measure of the accessibility of individual pores for two-phase flow relative to pores that contain no drops, and the relative velocity of the drop in the wavy-wall tube is intimately related to the relative mobility of the suspended phase in an actual porous structure, at least as reflected by data for the one tube which we have currently studied.

Although the results for drop deformation and breakup in the present work are mainly qualitative, they may be compared to previous results obtained for kinematically well-characterized flows. The problem of drop breakup in steady 
two-dimensional homogeneous flows has been treated by Taylor (1934) and Grace (1971, 1982), among others. Experiments show that breakup occurs for a critical shear rate which depends both on material properties and on the detailed kinematics of the flow field. Breakup in two-dimensional and axisymmetric elongational flows has been considered by Acrivos \& Lo (1978), Hinch \& Acrivos (1980) and Rallison \& Acrivos (1978). Theoretical results which encompass an arbitrary linear steady flow field have been obtained by Rallison (1981).

Grace has observed a transient mode of breakup which is particularly interesting here in view of the Lagrangian unsteadiness of our problem. Under some conditions, when a drop was sufficiently deformed in a steady two-dimensional elongation, breakup occurred after the flow was stopped, even though the drop continued to deform further without breaking if the flow was maintained. More recently, Han \& Funatsu (1978) studied the creeping motion of drops suspended in flow through a single, abrupt contraction, while Chin \& Han $(1979,1980)$ considered the related problem of flow of drops through a tapered tube. In both cases, breakup occurred downstream of the contraction, i.e. in a region of relaxation of the extensional deformation produced by the constriction. These results for homogeneous flows and for single contractions provide a basis for comparison with drop deformation in a periodic flow field.

\section{Experimental}

\subsection{Apparatus}

The basic apparatus described in (I) and (II) was modified for use in the present study. The current configuration is illustrated in figure 1.

Suspending fluid was pumped from a reservoir into a holding section which was immersed in a constant-temperature bath maintained at $25 \pm 0.1^{\circ} \mathrm{C}$. The suspending fluid then entered a $70 \mathrm{~cm}$ long straight circular entry section of internal diameter $0.90 \mathrm{~cm}$. This led to the test section, which was composed of eighteen individual periodic 'units' with the shape and dimensions illustrated in figure 2 . The crosssectional area of the tube varies by a factor of 3.2. Each unit was bored from a Lucite cylinder using a special tool, and the units were designed so that they could be attached and held in place under compression by two brackets. Three of the units were fitted with pressure ports at the point of minimum diameter. Another straight circular section of radius $0.45 \mathrm{~cm}$ and length $40 \mathrm{~cm}$ followed the test section. Relaxation of the drop shape was observed in this section.

The change in the pressure difference $\Delta P^{+}$due to the presence of the drop was measured directly, according to the method detailed in (I) and (II). In the previous studies, up to 24 drops were used to enhance the pressure signal. Here, however, the sensitivity of the transducer was increased and the metering pump improved so that pressure readings could be made for a single drop. This is essential, because, if more than one drop were used, the relative positions of the drops with respect to the period of the tube would constitute additional parameters for the experiment. The drops were injected using a micrometer syringe, and they attained an equilibrium position in the straight-wall entry section which was found to be independent of the details of the injection procedure.

The velocity $U$ of the drop was determined by measuring the elapsed time for the drop centre to move through a specified number of tube wavelengths.

The drops were photographed by a videocamera equipped with zoom and close-up lenses. The camera was mounted on a motorized horizontal rail system so that the 


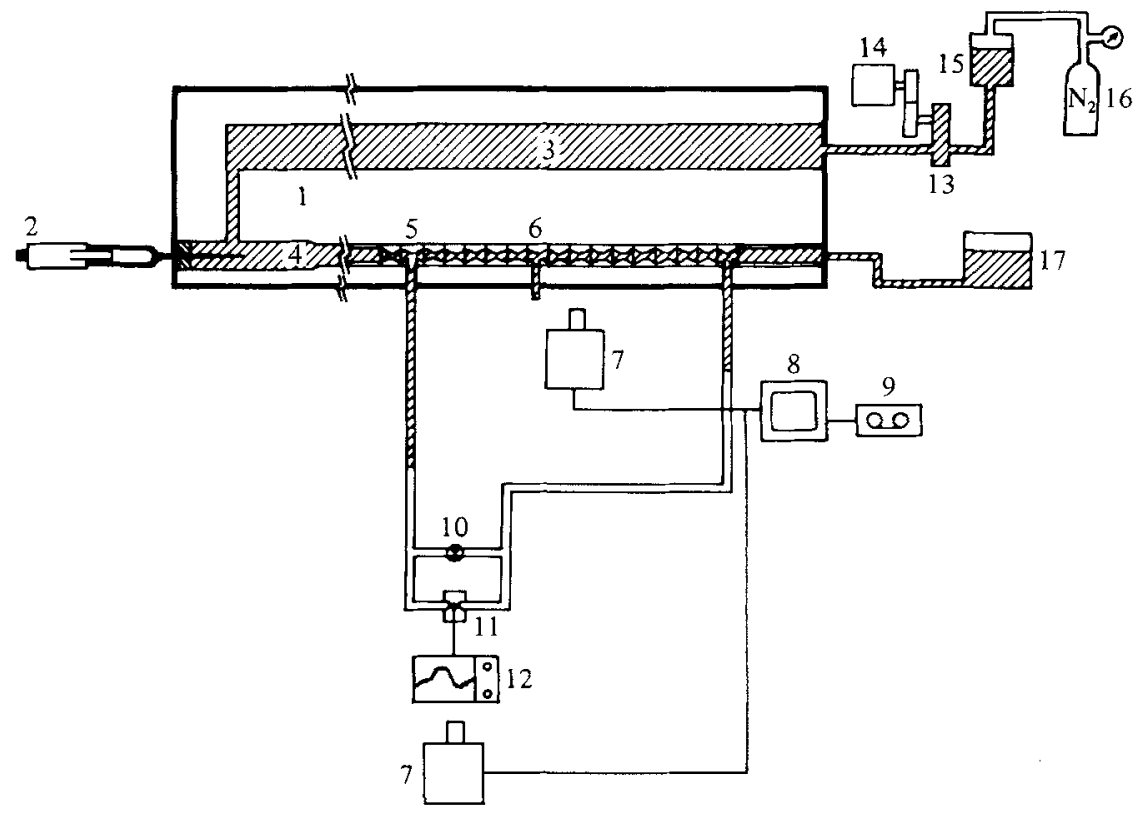

Figure 1. Schematic diagram of experimental set-up (not to scale): (1) constant-temperature bath; (2) micrometer syringe; (3) suspending-fluid storage section; (4) test section; (5) pressure port; (6) auxiliary pressure port; $(7)$ videocamera; $(8)$ videomonitor; $(9)$ videotape recorder; $(10)$ manometer bypass valve; (11) pressure transducer; (12) transducer indicator; (13) gear pump;(14) variable-speed motor; (15) pressurized suspending-fluid reservoir; (16) compressed nitrogen.

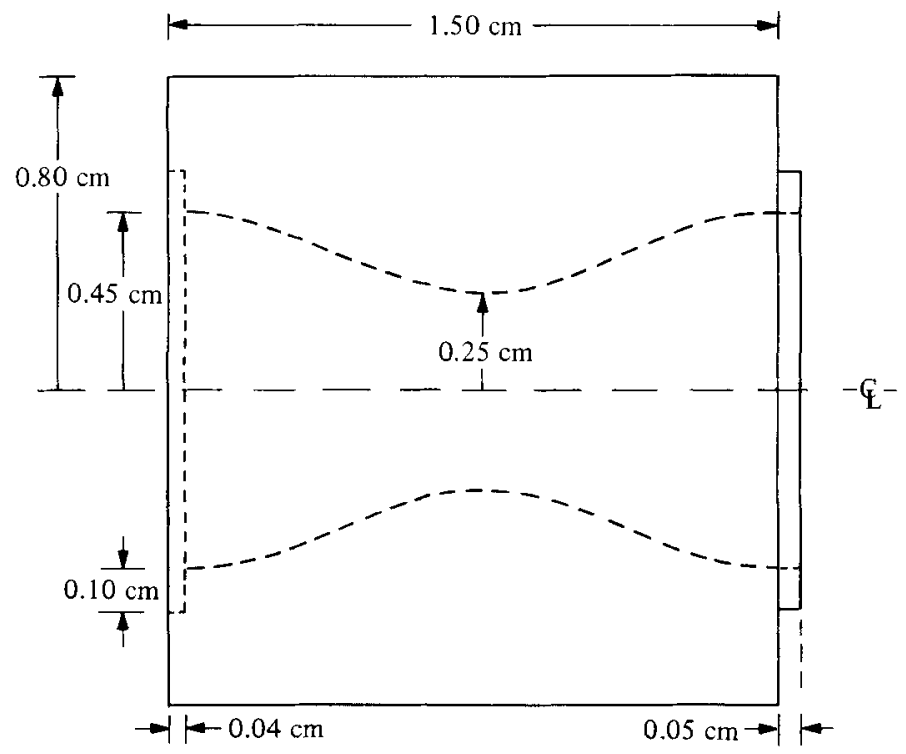

Figure 2. Scale drawing of a test-section unit.

drop could be tracked continuously as it passed through the test section. The output of the transducer indicator was also displayed on the video monitor. The combined images were recorded on videotape; thus a continuous record of the shape and position of the drop along with the simultaneous reading of the additional pressure drop was obtained. 


\subsection{Materials and parameters of the experiment}

Measurements were taken for 19 fluid systems encompassing a wide range of material parameters. Specifications for all systems are listed in table 1. The material and flow parameters that characterize each experiment are: $\sigma$, the viscosity of the drop $\mu_{i}$ relative to the viscosity of the suspending fluid $\mu_{0} ; \Delta \rho / \rho_{0}$, the difference in density between the drop and suspending fluid relative to that of the suspending fluid; $\lambda$, the undeformed drop radius divided by a characteristic tube radius; $\Gamma\left(=\mu_{0} V / \gamma\right)$, a capillary number, where $\gamma$ is the interfacial tension between the drop and suspending fluid and $V$ is the average flow velocity; and rheological properties of the suspending fluid. The experiments were all performed with the volumetric flow rate $Q$ held constant, while the contribution of the drop to the pressure difference varied with time. This is distinct from an associated problem where $\Delta P$, the total pressure drop, is held constant and the flow rate $Q$ varies with time owing to the presence of the suspended drop.

The viscoelastic suspending fluids were aqueous solutions of Dow Separan AP-30, a rheologically well-characterized polyacrylamide (Leal, Skoog \& Acrivos 1971). To study the effects of suspending fluid viscoelasticity, two polymer concentrations were used, $0.5 \%$ and $1.0 \%$ by weight.

Since the Separan solutions are shear-thinning, there is some arbitrariness in assigning a single value to the suspending fluid viscosity $\mu_{0}$ for the purposes of correlating data. In (I) and (II), the viscosity was evaluated at the wall shear rate, but the oscillatory tube radius adds the further complication that the wall shear rate varies with axial position. To maintain some comparison with the previous experiments, the sinusoidal geometry must be replaced by an 'average ' or 'effective' radius which is independent of axial position.

Data correlation will depend on how the effective radius is chosen, and Franzen (1979) has proposed several definitions, including one which is based on the assumption that the Hagen-Poiseuille law applies over an infinitesmal length $\mathrm{d} z$ everywhere along the tube axis. The same type of correlating parameter was considered by Sheffield \& Metzner (1976). The effective radius $r_{\mathrm{HP}}$ is then expressed as

$$
r_{\mathrm{HP}}=\left(\frac{L A_{0}^{2}}{\pi}\left[\int_{0}^{L} \frac{\mathrm{d} z}{\epsilon^{2}(z)}\right]^{-1}\right)^{\frac{1}{4}} .
$$

Here $\epsilon(z)$ is the function that describes the axial variation in tube cross-sectional area, $A_{0}$ is the area at $z=0$, and $L$ is the period of the tube-wall oscillation. For the present experiment, the tube radius $R(z)$ is given by $R=R_{0}+a \sin (2 \pi z / L)$, where $R_{0}=0.35 \mathrm{~cm}, a=0.1 \mathrm{~cm}$ and $L=1.50 \mathrm{~cm}$. The calculated value of $r_{\mathrm{HP}}$ is $0.316 \mathrm{~cm}$. It should be pointed out that the average value for the amplitude of the radius, $0.350 \mathrm{~cm}$, and the radius that gives the average cross-sectional area of the tube, $0.357 \mathrm{~cm}$, as well as other 'effective radii' defined by Franzen (1979), differ from $r_{\mathrm{HP}}$.

The wall shear rate was calculated for a circular tube of radius $r_{\mathrm{HP}}$ assuming that the fluids exhibit power-law dependence of the viscosity on the shear rate $\gamma$,

$$
\mu=m \gamma^{n-1} \text {. }
$$

The appropriate parameters are $n=0.45, m=12.0$ for $0.5 \%$ Separan and $n=0.40$, $m=33.8$ for $1.0 \%$ Separan (cf. Leal et al. 1971). The effective viscosity is then $\tau_{\mathrm{w}} / \beta_{\mathrm{w}}$ where the wall shear stress $\tau_{\mathbf{w}}$ is calculated from a force balance as

$$
\tau_{\mathrm{w}}=r_{\mathrm{HP}} \Delta P / 2 L,
$$




\begin{tabular}{|c|c|c|c|c|c|c|c|c|}
\hline \multirow[b]{2}{*}{ System } & \multirow[b]{2}{*}{ Suspending fluid } & \multirow[b]{2}{*}{ Drop fluid } & \multirow{2}{*}{$\begin{array}{c}\gamma \\
(\mathrm{dyn} / \mathrm{cm})\end{array}$} & \multirow[b]{2}{*}{$\Delta \rho / \rho_{0}$} & \multicolumn{2}{|c|}{$V=0.24$} & \multicolumn{2}{|c|}{$V=0.46 \mathrm{~cm} / \mathrm{s}$} \\
\hline & & & & & $\sigma$ & $\Gamma$ & $\sigma$ & $\Gamma$ \\
\hline & Newtonian: & Newtonian: & & & & & & \\
\hline 1 & UCON LB-1715 & Water & 3.5 & 0.000 & 0.0014 & 0.50 & 0.0014 & 0.94 \\
\hline $2 a$ & $95.45 \%$ glycerol & $\mathrm{DC} 200+\mathrm{CCl}_{4}$ & 22.0 & 0.003 & 0.40 & 0.043 & 0.40 & 0.083 \\
\hline $2 b$ & $\mathbf{9 5 . 4 5} \%$ glycerol & $\mathrm{DC} 200+\mathrm{CCl}_{4}$ & 22.0 & 0.000 & 0.40 & 0.043 & 0.40 & 0.083 \\
\hline $2 \mathrm{c}$ & $95.45 \%$ glycerol & $\mathrm{DC} 200+\mathrm{CCl}_{4}$ & 22.0 & 0.001 & 0.40 & 0.043 & 0.40 & 0.083 \\
\hline 3 & $95.45 \%$ glycerol & $\mathrm{DC} 200+\mathrm{CCl}_{4}$ & 22.0 & 0.000 & 0.60 & 0.043 & 0.60 & 0.083 \\
\hline 4 & $95.45 \%$ glycerol & DC510 & 1.3 & 0.000 & 0.70 & 1.34 & 0.70 & 2.54 \\
\hline 5 & $95.45 \%$ glycerol & DC510 & 1.3 & 0.000 & 7.52 & 1.34 & 7.52 & 2.54 \\
\hline 6 & $\mathbf{9 5 . 4 5} \%$ glycerol & DC510 & 1.3 & 0.000 & 14.6 & 1.34 & 14.6 & 2.54 \\
\hline 7 & UCON-LB 1715 & DC200 & 2.5 & 0.024 & 0.70 & 0.70 & 0.70 & 1.31 \\
\hline 8 & UCON-LB 1715 & DC200 & 2.5 & 0.022 & 17.9 & 0.70 & 17.9 & 1.31 \\
\hline & Viscoelastic: & Newtonian: & & & & & & \\
\hline 9 & $\mathbf{0 . 5} \%$ Separan & UCON LB & 5.3 & 0.000 & 0.20 & 0.34 & 0.30 & 0.43 \\
\hline 10 & $0.5 \%$ Separan & UCON LB-X & 5.3 & 0.000 & 0.94 & 0.34 & 1.42 & 0.43 \\
\hline 11 & $0.5 \%$ Separan & DC510 & 18.5 & 0.000 & 12.6 & 0.073 & 18.2 & 0.096 \\
\hline 12 & $0.5 \%$ Separan & DC200 & 24.5 & 0.037 & 0.29 & 0.050 & 0.42 & 0.066 \\
\hline 13 & $0.5 \%$ Separan & DC200 & 18.1 & 0.036 & 16.1 & 0.069 & 23.2 & 0.091 \\
\hline 14 & $0.5 \%$ Separan & UCON LB & 5.0 & 0.026 & 0.10 & 0.24 & 0.14 & 0.33 \\
\hline 15 & $1.0 \%$ Separan & UCON LB-X & 5.1 & 0.000 & 0.081 & 0.86 & 0.12 & 1.10 \\
\hline 16 & $1.0 \%$ Separan & UCON LB-X & 5.1 & 0.000 & 0.38 & 0.89 & 0.57 & 1.12 \\
\hline 17 & $1.0 \%$ Separan & DC510 & 16.0 & 0.000 & 3.63 & 0.29 & 5.46 & 0.37 \\
\hline 18 & $1.0 \%$ Separan & DC200 & 21.7 & 0.039 & 0.085 & 0.19 & 0.13 & 0.24 \\
\hline 19 & $1.0 \%$ Separan & DC200 & 16.1 & 0.038 & 4.77 & 0.26 & 7.17 & 0.33 \\
\hline
\end{tabular}




\begin{tabular}{|c|c|c|c|c|c|c|}
\hline \multirow[b]{2}{*}{ Suspending fluid } & \multicolumn{3}{|c|}{$V=0.24 \mathrm{~cm} / \mathrm{s}$} & \multicolumn{3}{|c|}{$V=0.46 \mathrm{~cm} / \mathrm{s}$} \\
\hline & $\underset{\left(\mathbf{s}^{-1}\right)}{\beta_{\mathrm{w}}}$ & $\begin{array}{l}\mu_{0} \\
(\mathrm{P})\end{array}$ & $\begin{array}{c}N_{1} \\
\left(\mathrm{dyn} / \mathrm{em}^{2}\right)\end{array}$ & $\underset{\left(\mathrm{s}^{-1}\right)}{\beta_{\mathrm{w}}}$ & $\begin{array}{l}\mu_{0} \\
(\mathrm{P})\end{array}$ & $\begin{array}{c}N_{1} \\
\left(\mathrm{dyn} / \mathrm{cm}^{2}\right)\end{array}$ \\
\hline $\mathbf{9 5 . 4 5} \%$ glycerol & 3.09 & 3.94 & 0 & 5.81 & 3.94 & 0 \\
\hline UCON LB-1715 & 3.09 & 7.17 & 0 & 5.81 & 7.17 & 0 \\
\hline $0.5 \%$ Separan & 4.03 & 7.48 & 35 & 7.66 & 4.95 & 45 \\
\hline $1.0 \%$ Separan & 4.36 & 18.39 & 55 & 8.25 & 12.31 & 85 \\
\hline
\end{tabular}

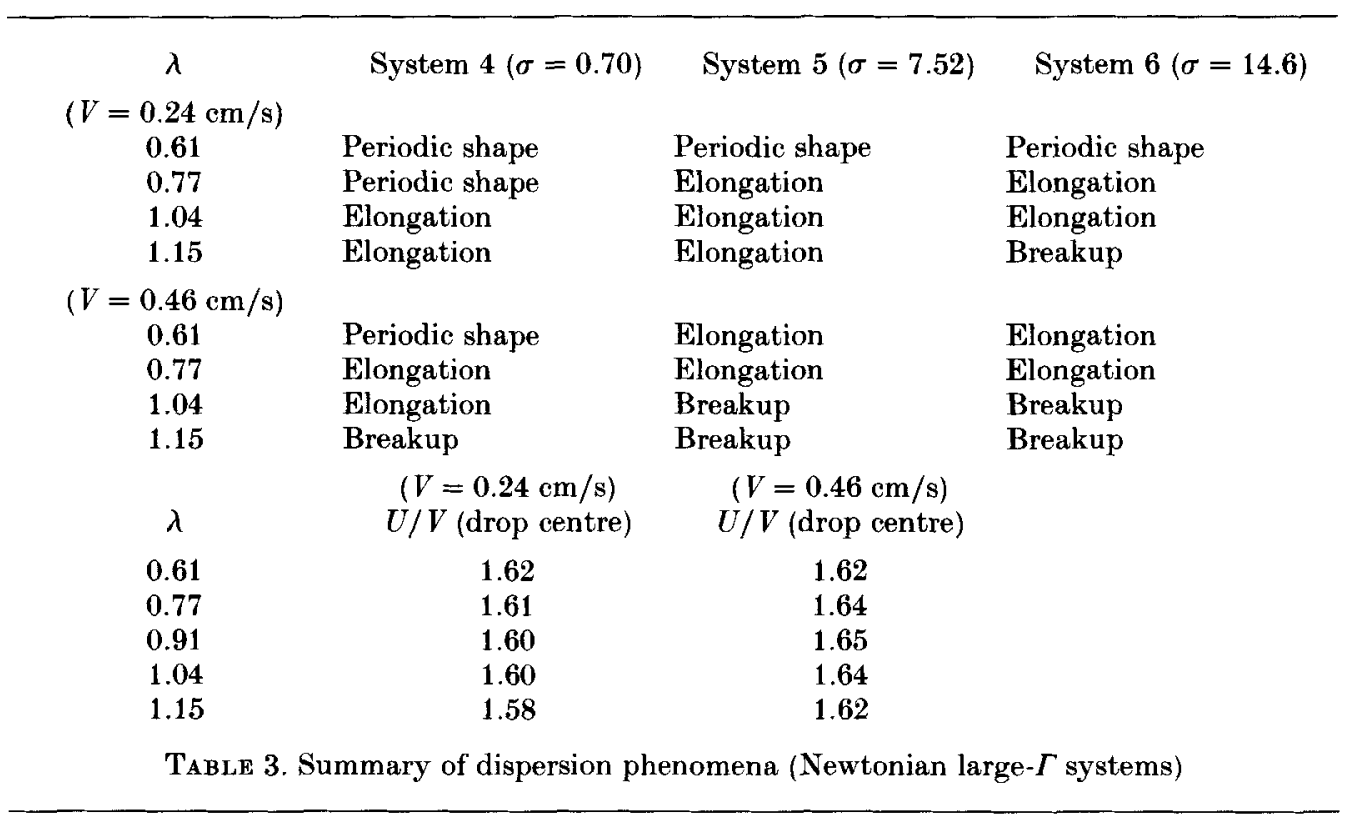

and the wall shear rate is

$$
\beta_{\mathrm{w}}=\frac{3 n+1}{4 n} \frac{4 Q}{\pi r_{\mathrm{HP}}^{3}}
$$

It was found that the value of the viscoelastic fluid 'viscosity' depended on the method of solution preparation; although care was taken to treat each solution identically, the value obtained for $\mu_{0}$ varied by as much as $15 \%$ for different preparations. Typical values for the suspending fluid properties are given in table 2 .

The (Newtonian) drop fluids consisted of various grades of UCON LB-Series lubricant, Dow Corning DC200, and Dow Corning DC510 silicone oils. Since $\Delta \rho / \rho_{0}$ is a parameter of the present experiments, densities were adjusted using clove oil with the UCON oils and carbon tetrachloride with the silicone oils.

\subsection{Conditions of the experiments}

The experiments were conducted at two volumetric flow rates, $Q=4.6$ and $8.7 \mathrm{ml} / \mathrm{min}$. Measurements of $\Delta P^{+}, U / V$, and drop shape were made for five drop sizes listed in table 3 . The average velocity $V$ was calculated by dividing the (known) volumetric flow rate $Q$ by the effective cross-sectional area $\pi r_{\mathrm{HP}}^{2}$, so that $V=Q / \pi r_{\mathrm{HP}}^{2}$. 
It may be noted, in this regard, that tracer particles were used to determine that no visible flow separation occurred during the course of the experiments. The nondimensional drop size $\lambda$ also is based on the effective Hagen-Poiseuille radius $r_{\mathrm{HP}}$, and this provides a direct comparison with the results of (I) and (II). The dimensionlesss extra pressure drop is defined as $\Delta P^{+} r_{\mathrm{HP}} / \mu_{0} V$.

\section{Pressure drop-flow rate characteristics for single-phase flow}

Before considering the full two-phase problem, we report pressure drop-flow rate data for the flow of the suspending fluid alone, i.e. with no drops present. For the New tonian fluids, we will show that the data are usefully correlated using the effective radius $r_{\text {HP }}$. For the viscoelastic fluids, these data indicate, at least qualitatively, the importance of shear-thinning and elastic behaviour in a wavy-wall tube under conditions covered in this experiment.

\subsection{Newtonian fluids}

Pressure drop-flow rate data for single-phase flow through conduits are usually correlated in terms of a friction factor and Reynolds number. For flow of a Newtonian fluid through a straight-wall circular tube, the friction factor can be defined by $f=r \Delta P / l \rho_{0} V^{2}$, where $r$ is the (constant) tube radius, $l$ is the tube length and $V$ is the average velocity. For laminar flow, the Hagen-Poiseuille law is then expressed as $f=16 / R e$. Provided that the effective tube radius $r_{\mathrm{HP}}$ is appropriate as a lengthscale for purposes of data correlation in a wavy-wall tube under conditions of the present experiments, the same relationship should hold between $f$ and $R e$ defined in terms of $r_{\text {HP. }}$. Data for the wavy-wall tube expressed in terms of $f$ and Re are shown in figure 3 ; they coincide precisely with the Hagen-Poiseuille law for a straight-wall tube with radius $r_{\mathbf{H P}}$, thus demonstrating the relevance of $r_{\mathbf{H P}}$ as a lengthscale of the flow under present conditions.

Detailed velocity and pressure fields for flow through periodically constricted tubes of a variety of shapes have been calculated by Payatakes, Tien \& Turian (1973), Payatakes \& Neira (1977), Neira \& Payatakes (1979), Fedkiw \& Newman (1977), Deiber \& Schowalter (1979) and Payatakes \& Tilton (1983). These results consistently show that a log-log plot of an appropriately defined friction factor versus $R e$ yields a linear relationship as shown in figure 3 , at least up to the value of $R e$ at which either flow separation or turbulence occurs. We have compared quantitatively our pressure drop-flow rate data with the numerical calculations of Payatakes \& Tilton (1983) for flow of a Newtonian fluid through a tube of sinusoidally varying diameter. The calculated values for $f$ as a function of $R e$ are within $4 \%$ of our measured values over the range of $R e$ covered in this experiment.

\subsection{Non-Newtonian fluids}

Pressure drop data for viscoelastic fluids in a straight-wall tube have been shown by many authors to follow the Hagen-Poiseuille law provided that a generalized Reynolds number is employed which incorporates the power-law parameters $m$ and $n$ (equation (2)) in the form

$$
R e_{n}=2^{3-n}\left(\frac{3 n+1}{n}\right)^{-n} \frac{(2 r)^{n} V^{2-n}}{m} .
$$

Although other manifestations of viscoelasticity, such as normal-stress effects and relaxation phenomena associated with fluid elasticity, will presumably be significant 


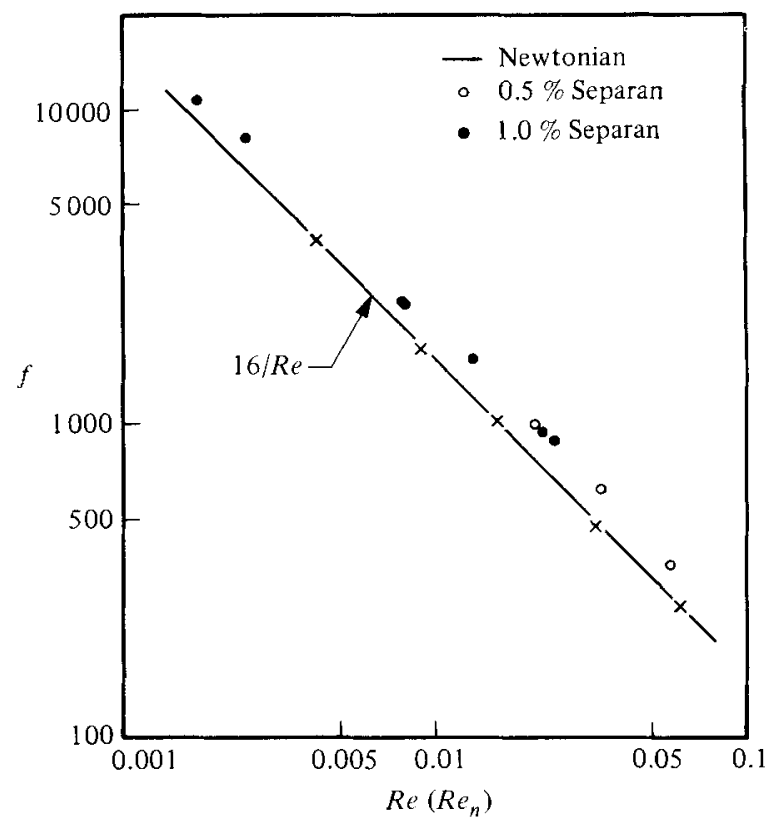

Figure 3. Friction factor $f v s$. Re for Newtonian and viscoelastic flow; $R e_{n}$ is defined in terms of power-law parameters: $x$, Newtonian data.

under some conditions in the wavy-wall tube, the results of $\$ 3.1$ on Newtonian fluids suggest that these effects should be manifested by systematic deviations from the power-law fluid correlation. Experimental observations of flow of polymer solutions through a tube with abrupt alternate expansions and contractions by Michele (1977), of flow through several periodic tube geometries by Franzen (1979), and of flow through granular beds, packed beds and bundles of capillary tubes by Marshall \& Metzner (1967), Savins (1969), James \& McLaren (1975) and Elata et al. (1977), suggest that departures (sometimes dramatic) from a linear relationship between $f$ and $R e$, which takes into account shear-thinning of the fluid and the physical structure of the porous matrix, occur when the characteristic relaxation time of the fluid is comparable to the characteristic convective time; then, apparently, dynamies of the flow are governed, in part, by the elastic response of the fluid. A convenient measure of the intrinsic relaxation time for the fluid relative to the characteristic timescale for Lagrangian unsteady flow is the Deborah number.

Data for the $0.5 \%$ and $1.0 \%$ aqueous solutions of Separan are shown in figure 3 . The power-law model parameters were evaluated from simple shear flow data over a range of shear rates $0.3<\gamma<10 \mathrm{~s}^{-1}$. It can be seen from table 2 that this range of $\gamma$ encompasses the values of the wall shear rate $\beta_{\mathrm{w}}$ (based on $r_{\mathrm{HP}}$ ) appropriate to the experiment. It is clear from figure 3 that the measured value of $f$ for each of the viscoelastic cases is larger than $f$ for either a power-law or Newtonian fluid at the identical value of Re. 'The data exceed the 'power-law' prediction in all cases and by as much as $35 \%$. Similar deviations, both in direction and magnitude, were observed by both Michele (1977) and Franzen (1979).

It is of interest to verify that the deviations from the power-law friction-factor correlation are, in fact, a consequence of viscoelasticity rather, for example, than an indication that $r_{\mathrm{HP}}$ (and thus $V$ ) is inappropriate as a characteristie lengthseale for the non-Newtonian fluid case. The most direct indication of this fact is to show that 


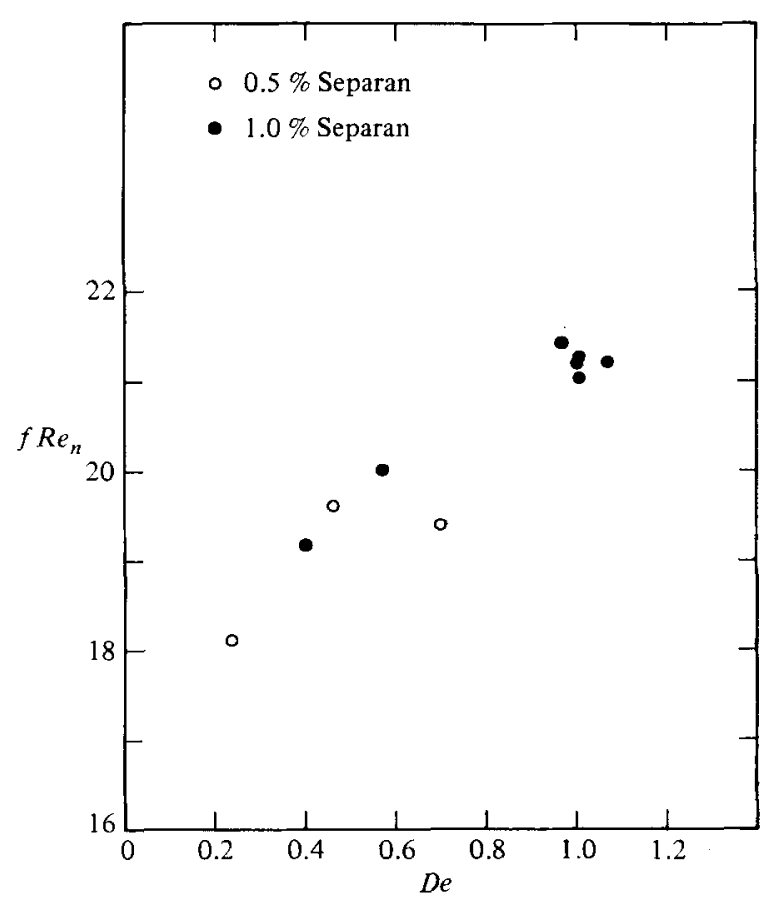

Figure 4. Data for $f R e_{n}$ as a function of Deborah number $D e$ for the viscoelastic fluids.

the experimental deviations are correlated with an appropriately chosen Deborah number. In order to estimate $D e$ for the present experiment we have used the results of Marshall \& Metzner (1967). Flow through a convergent section of the tube is, in effect, modelled by flow toward the apex of a circular cone. Using this simple geometry for the flow channel, Marshall \& Metzner calculated a timescale over which the deformation rate varies, and hence an expression for $D e$ :

$$
D e=\theta \frac{V}{L_{1}}\left(\frac{3}{2}+\frac{1}{4}\left(\frac{L_{1}}{L_{2}}\right)^{2}\right)^{\frac{1}{4}}
$$

where $V$ is the nominal velocity, $\theta$ is the characteristic relaxation time of the fluid, and $L_{1}$ and $L_{2}$ are the characteristic length and width of the cone. To estimate $\theta$ for the Separan solutions, we have used the contravariant form of the convected Maxwell model which relates $\theta$ to the normal stress measured in steady simple shear flow. The value of $\theta$ is then given by

$$
\theta=\frac{1}{2 \dot{\gamma}^{2}}\left(\frac{\tau_{11}-\tau_{22}}{\mu_{0}(\dot{\gamma})}\right),
$$

where $\tau_{11}-\tau_{22}$ is the primary normal stress difference, $\mu_{0}$ is the viscosity and $\dot{\gamma}$ is the shear rate. Unfortunately, the characteristic time of the fluid estimated in this manner is a function of the shear rate $\dot{\gamma}$. This fact is at least partly responsible for wide variations in the estimated value of $D e$ necessary for onset of elastic behaviour in different experiments. We have evaluated $\theta$ at the shear rate contained in (6) to determine $D e$. The product of $f R e_{n}$ for each point in figure 3 is plotted as a function of $D e$ in figure 4. The data plotted in this fashion show a systematic increase in the deviation from 'power-law' (or purely viscous) fluid behaviour with increase of $D e$, as expected. Similar experimental results were obtained by Michele (1977), Franzen 
(1979) and Deiber \& Schowalter (1981) for viscoelastic flow through a variety of oscillatory circular tubes for comparable values of $D e$.

These experiments on the motion of a single-phase fluid through the wavy-wall tube serve primarily to verify the relevance of the effective tube radius $r_{\mathrm{HP}}$, and quantities derived from $r_{\text {HP }}$, such as the characteristic velocity $V$ for purposes of data correlation under conditions of the present experiments. Let us now turn to the primary subject of our paper, the motion of suspended large drops through the wavy-wall tube.

\section{Experimental results and discussion for two-phase flow}

As a suspended drop moves through the wavy-wall tube, it causes a periodic variation in the pressure drop. Each oscillation of the output signal corresponds to the drop's passage through one period of the oscillatory test section. Spurious transient disturbances, which occur at the beginning and end of each experiment, are caused by the drop's passage over the pressure ports.

The pressure signature for each case depends on the detailed time-dependent drop shape, which, in turn, depends upon all the dimensionless groups that characterize each experiment. We will discuss the effect of these parameters shortly when we consider special cases. However, the details aside, our experiments show that the gross shape of the drop seems to depend most strongly on the value of the capillary number $\Gamma$, at least for the range of parameters covered here. As the value of $\Gamma$ varies over two orders of magnitude, and all other parameters remain fixed, we observe radically different drop shapes. It is apparent that $\Gamma$, which is the ratio of viscous forces to interfacial tension forces, is the parameter which distinguishes between cases of large deformation and small deformation, and even between breakup and no breakup. Therefore, we find it useful to organize our results according to the magnitude of $\Gamma$ and to discuss separately the effects of the remaining parameters for each case. Our experiment pertains to the motion of droplets under most favourable conditions with respect to oil-recovery processes, since the values of $\Gamma$ covered here are appropriate for recovery where a significant reduction in interfacial tension has been accomplished.

In order to present data economically, we show the dimensionless additional pressure drop, which will be denoted in this section as simply $\Delta P^{+}$, and the relative velocity $U / V$ as a function of dimensionless drop size $\lambda$. The value of $\Delta P^{+}$in the figures which follow is an arithmetic average of the maximum and minimum amplitudes of the periodic pressure signal. We also show the non-dimensionalized magnitude of the periodic part of the signal $\delta\left(\Delta P^{+}\right)=\Delta P_{\max }^{+}-\Delta P_{\min }^{+}$, as a function of $\lambda$. The values of $U / V$ reported in the figures represent averages, too, in so far as $U$ is the velocity of the centre of the drop averaged over many tube wall oscillations, and $V$ is an average or bulk velocity of the flow. The measured values of $\sigma, \Gamma$ and $\Delta \rho / \rho_{0}$ for each fluid system and for each flow rate are indicated on the figures and also compiled in table 1.

\subsubsection{Small- $\Gamma$ systems}

\subsection{Newtonian fluid systems}

We consider first the results for systems $2 a, 2 b, 2 c$, and 3 , which have $\Gamma<0.083$. This small- $\Gamma$ group contains the 'least deformable' drops, and there is no drop breakup for any case in this group.

Figure 5 shows the pressure signal and the corresponding drop shape for several positions spanning one tube period for system $2 b$ with $\lambda=0.76,0.91$, and 1.15 , and 

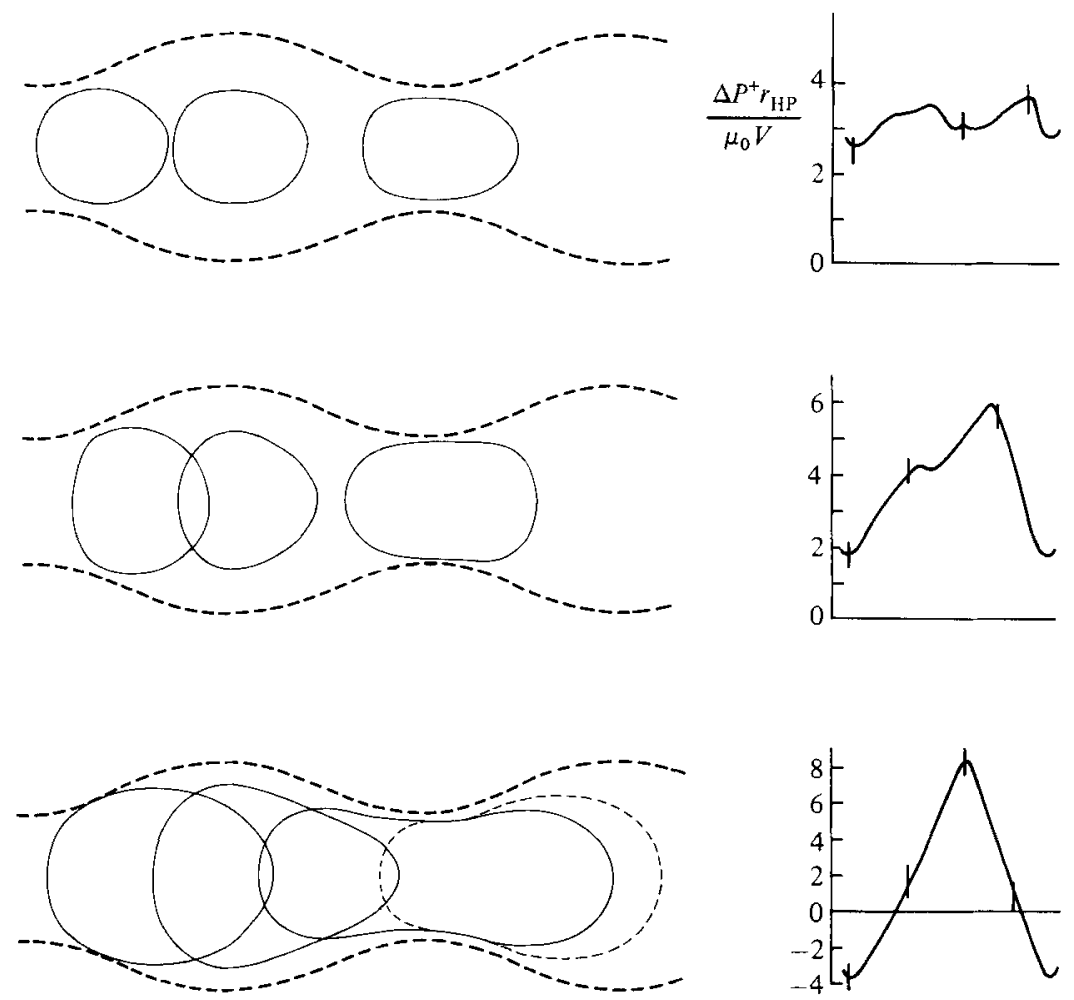

Figure 5. Typical drop shapes (drawn from videorecordings) and the corresponding dimensionless additional pressure difference for a system $2 b$ drop $(\Gamma=0.043$, Newtonian suspending fluid) as it moves through one period of the wavy-wall tube. The flow is from left to right. The tick marks on the pressure curve correspond to the values obtained at the instant when the drop exhibits the illustrated shapes, from left to right. The cases shown are for three drop sizes: $\lambda=0.76,0.91$ and 1.15 .

for $\Gamma=0.043$. The drop deforms to 'squeeze through' the throat of the tube and then follows the tube wall contour closely as it emerges into the wider section of the tube. The maximum deformation occurs when the drop is approximately midway through the cross-section of minimum area, and this coincides with the maximum value of $\Delta P^{+}$. The drop shape at this specific point is illustrated for each case in the figure. The gap between the drop and the tube wall at this instant is small for these small- $\Gamma$ systems. For sufficiently small drops, $\lambda<0.7$ (not shown in figure 5 ) the undeformed diameter of the drop is smaller than the minimum diameter of the tube and, in these cases, the magnitude of $\delta\left(\Delta P^{+}\right)$is less than the uncertainty of the measurement. For $\lambda>0.7$, the magnitude of the periodic part of the signal increases rapidly with $\lambda$, and the pressure signal for the larger drops becomes nearly sinusoidal in shape.

Figure 6 shows the average of these signals, $\Delta P^{+}$, and the magnitude of the periodic part, $\delta\left(\Delta P^{+}\right)$, as a function of drop size $\lambda$ for selected small- $\Gamma$ systems. When average values are considered, the additional pressure drop shows a trend which is reminiscent of the behaviour for drops with $\sigma<1$ in the straight-wall tube flow. For comparison purposes, results for system $3 a$ of $(I)(\sigma=0.58, \Gamma=0.075)$, which is comparable to system 3 in the present experiment, are shown in figure 6 . Although the value of $\Delta P^{+}$ is positive for small drops, it eventually attains a maximum and ultimately becomes negative as the drop is made sufficiently large. It was shown in $(I)$ that the value of $\Delta P^{+}$is deterimined by several competing mechanisms: the disturbance of the flow 


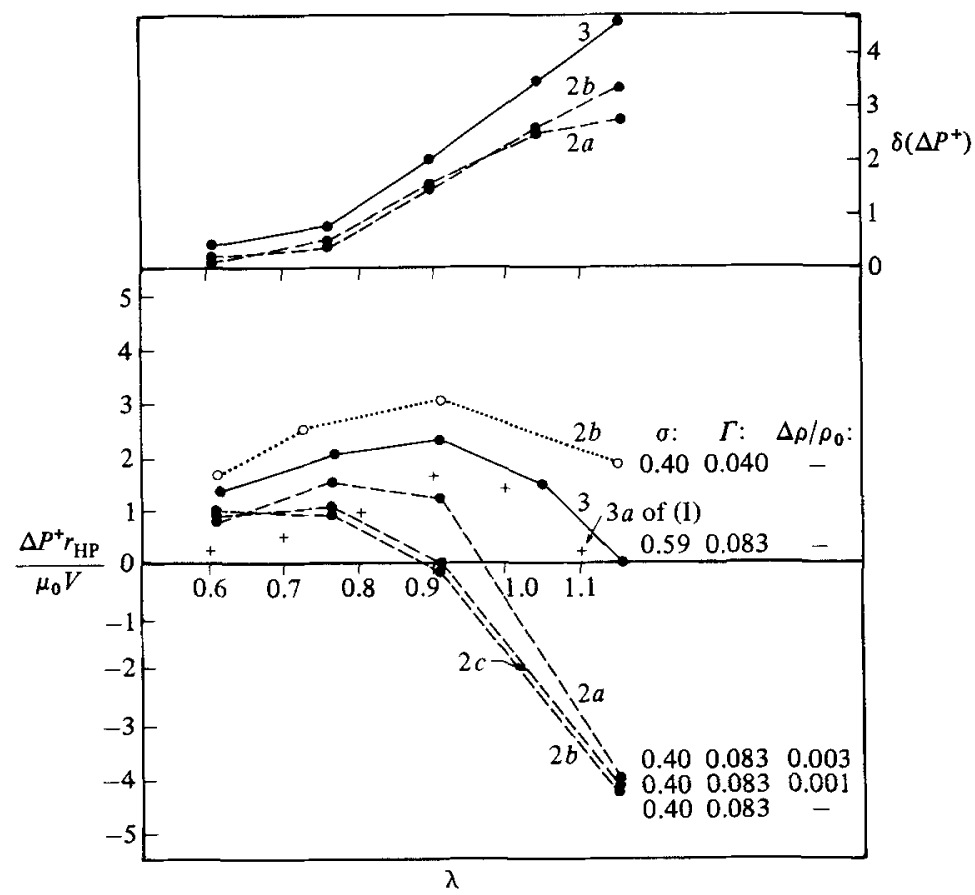

Figure 6. Dimensionless average additional pressure drop and dimensionless magnitude of the oscillatory part as a function of dimensionless drop size for small- $\Gamma$ systems in a Newtonian suspending fluid. System $2 b$ values for $\delta\left(\Delta P^{+}\right)$for $\Gamma=0.043$ (not shown) increase monotonically with $\lambda$ from 0.8 to 12.1 . ' $2 b$ ' indicates system $2 b$, etc. + , system $3 a$ of (I) $(\sigma=0.58, \Gamma=0.075$ ).

caused by the drop, drop deformation, interactions between the drop and wall, and the simple replacement of suspending fluid with drop fluid of a different viscosity. Results from (I) indicate that, when the drop is made sufficiently large, the contribution from simple replacement of fluid determines the sign of $\Delta P^{+}$, and the identical large- $\lambda$ asymptote for the average additional pressure drop is observed in the present experiments.

However, when the instantaneous value of the additional pressure drop is considered, the influence of the tube geometry is exposed. In particular, the time-dependent additional pressure drop in the wavy-wall tube experiment cannot be deduced from the data for the straight-wall tube by simply accounting for the local variation of relative drop size as the drop moves through a period of the tube. Indeed, results from figure 6 indicate that the maximum value of the additional pressure drop which is attained when the drop squeezes through the tube throat exceeds the values of $\Delta P^{+}$found for a corresponding fluid system in the straight-wall tube for any value of $\lambda$. This means that, as the drop moves through the throat of the wavy-wall tube, it causes an additional pressure drop which is larger than that which would occur for the same drop in a straight-wall tube with the throat diameter.

The qualitative effect of flow rate on the additional pressure drop and on the relative velocity is similar to the two flows. Figure 6 shows that the average value of the dimensionless additional pressure drop decreases as the flow rate is made larger, and the same result was found for the straight-wall tube. There, it is a consequence of increased drop deformation for larger $V$ (i.e. larger $I$ ), and this qualitative explanation also seems appropriate here. For the same reason, the 'average' mobility $U / V$ of the drop increases with $V$. The value of $U / V$ for system $2 b$ drops for the 


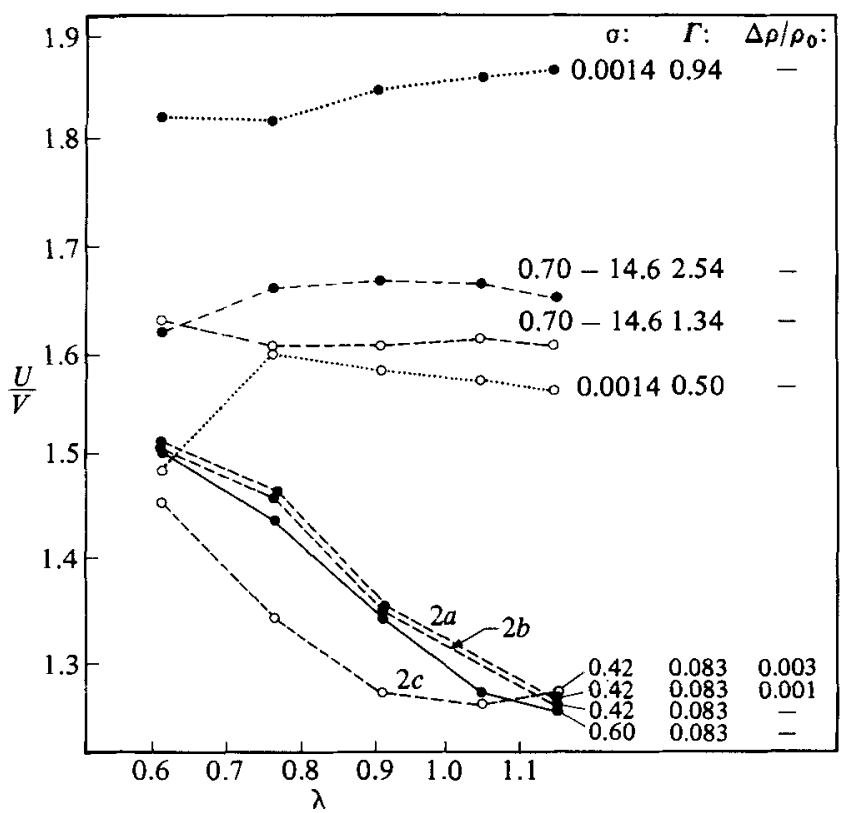

Figure 7. Relative drop velocity as a function of dimensionless drop size for all systems with a Newtonian suspending fluid. System $2 c$ for $\Gamma=0.043$ is not shown (see text).
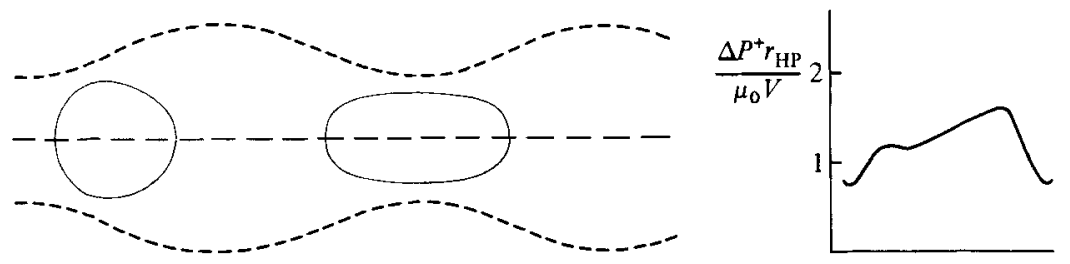

Figure 8. Typical drop shapes and the corresponding dimensionless additional pressure difference for a system $2 b$ drop $(\Gamma=0.083$, Newtonian suspending fluid) with $\lambda=0.76$.

smaller flow rate, i.e. $\Gamma=0.043$, are $1.36,1.20$ and 1.12 for $\lambda=0.62,0.91$ and 1.15 respectively. The corresponding values shown in figure 7 for the larger flow rate, i.e. $\Gamma=0.083$, are $1.50,1.36$ and 1.28 . However, the role of drop deformation is even more evident in the instantaneous value of the pressure drop, which is reflected in the magnitude of the periodic part of the pressure signal. Figure 6 shows that the value of $\delta\left(\Delta P^{+}\right)$for system $2 b$ with $\Gamma=0.083$ increases with $\lambda$ from $\approx 0.2$ to $\approx 3.3$ over the range of $\lambda$ studied; however, the corresponding data for $\delta\left(\Delta P^{+}\right.$) when $\Gamma=0.043$ (not shown in figure 7) range from 0.8 to 12.1 over the same range of $\lambda$. Similar trends are observed for the other small- $\Gamma$ systems. Figure 8 shows the drop shape and corresponding pressure signature for the same fluid system illustrated in figure 5 , but for the larger flow rate. It is clear that the degree of drop deformation increases with $V$, and this is especially apparent in the narrow tube throat. The gap width between the drop and the tube wall in the constriction increases with $V$. It was shown in (I) and (II) that these thin regions between the drop surface and the tube wall can act as lubrication layers, and hence it is not surprising that our results are highly sensitive to the degree of deformation and the corresponding gap thickness.

The effects of the remaining parameters, viz the viscosity ratio $\sigma$ and the nonneutral buoyancy parameter $\Delta \rho / \rho_{0}$, are seen mainly in the average value of the 


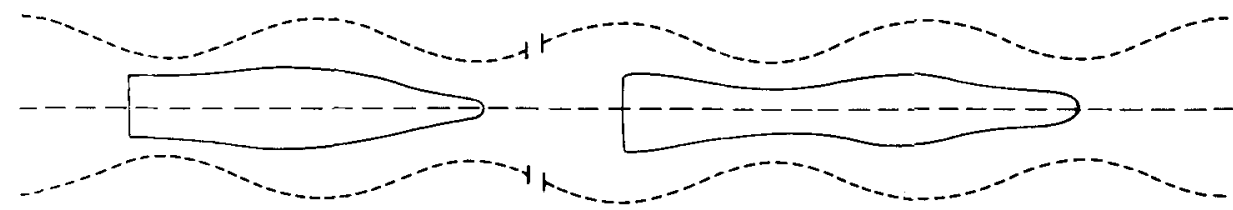

(a)

(b)

Figure 9. System 4 drop ( $\Gamma=1.34$, Newtonian suspending fluid) with $\lambda=1.04$ at two locations in the wavy-wall test section (see text).

pressure signal $\Delta P^{+}$. Figures 6 and 7 show that the effects of an increased viscosity relative to the viscosity of the suspending fluid are a larger value of $\Delta P^{+}$and a small decrease in $U / V$. Furthermore, the value of the periodic part $\delta\left(\Delta P^{+}\right)$increases slightly with $\sigma$, and this corresponds to small changes in the drop shape, especially in the tube throat. The averaged values of $\Delta P^{+}$and $U / V$ are sensitive to extremely small values of $\Delta \rho / \rho_{0}(<0.003)$, even though the magnitude of the periodic part of the pressure signal remains unchanged. It can be seen, though, that the sensitivity to drop eccentricity diminishes as $\lambda \rightarrow 1$, which also occurs for the straight-wall tube. In every case studied, when the drop practically fills the tube, the effect of a slight degree of asymmetry both in position and in shape are unimportant. Furthermore, we observe that a small degree of non-neutral buoyancy does not significantly affect the shape of large drops, especially in the tube throat, which explains the relative insensitivity of $\delta\left(\Delta P^{+}\right)$to the value of $\Delta \rho / \rho_{0}$.

\subsubsection{Large- $\Gamma$ systems}

For system 1 and systems $4-8$, the value of the capillary number exceeds 0.5 , and therefore we expect a greater degree of deformation for these systems than that reported for the small- $\Gamma$ systems in $\$ 4.1 .1$. Indeed, for the large- $\Gamma$ systems, we observe two modes of drop deformation and breakup, and these modes are distinguished by the value of the viscosity ratio $\sigma: \sigma \approx 1$ and $\sigma \ll 1$.

For $\sigma \approx 1$, a small drop $(\lambda<0.7)$ exhibits periodic deformation, although, at any instant, the degree of deformation is considerably greater than for the corresponding small- $\Gamma$ cases. However, a sufficiently large drop experiences a 'permanent' elongation along the axis of the wavy-wall tube. The length of the drop increases relatively slowly over the first five or six tube-wall oscillations, but the rate of stretching abruptly increases when the drop becomes sufficiently long that the leading edge of the drop invades the next constriction before the trailing edge has completely evacuated the preceding one. The situation is illustrated schematically in figure 9, where a system 4 drop with $\lambda=1.04$ is shown at two locations in the wavy-wall tube. Figure $9(a)$ shows the drop as the leading edge penetrates the second throat of the wavy-wall test section. At this instant, the trailing portion of the drop has, for the most part, passed through the previous throat. On the other hand, figure $9(b)$ shows the same drop as the leading edge invades the eighth constriction of the test section; here the trailing edge has not evacuated the previous throat. Thus the rate of increase in drop length is considerably greater in the configuration shown in figure $9(b)$. Elongation continues until an equilibrium shape is attained, except for the largest drops which exit the test section before reaching a steady shape. The drops can eventually form strands or filaments, and closely resemble mobilized strips of oil photographed by Wasan et al. (1979) during a surfactant flood of an actual porous structure. In the present experiment, the strands always remain stable while in the wavy-wall test section, but when sufficiently elongated, they break up in the straight-wall relaxation 


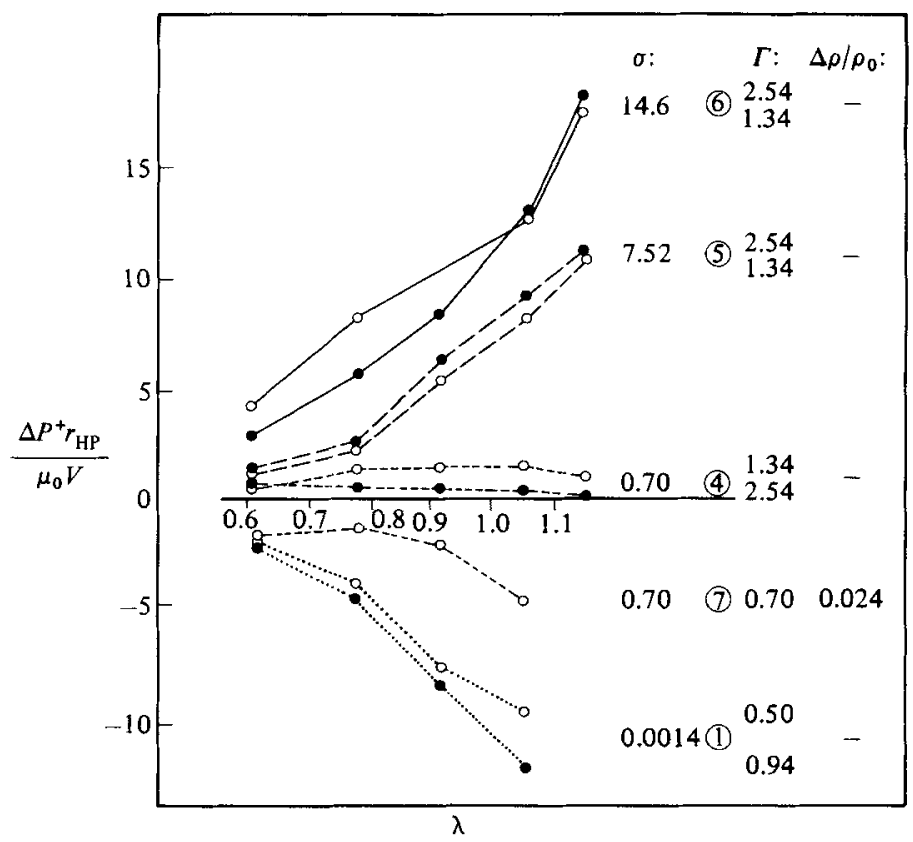

Figure 10. Dimensionless average additional pressure drop as a function of dimensionless drop size for large- $\Gamma$ systems, Newtonian suspending fluid.

section and form up to five roughly equal-sized smaller droplets. It is apparent that strand formation occurs for a given suspending fluid when the timescale associated with drop deformation is greater than the characteristic timescale of the periodic flow, or

$$
\frac{\lambda r_{\mathrm{HP}} \mu_{\mathrm{i}}}{\gamma}>\frac{L}{U}
$$

where $\mu_{\mathrm{i}}$ is the drop viscosity. The effect of an increase in $V$ (i.e. $\Gamma$ ) is to shift the required drop size for strand formation and breakup to smaller values, and this is summarized in table 3 . The data show also that increased values of the drop viscosity and decreased values of the interfacial tension tend to enhance the formation of strands, in qualitative accord with the above inequality.

The effect of an eccentric position for the drop with $\sigma \approx 1$ was tested with systems 7 and 8 , where $\Delta \rho / \rho_{0}$ was 0.022 and 0.024 respectively. Under every set of conditions tested, the non-neutrally buoyant drops undergo greater deformation than their neutrally buoyant counterparts. The drops deviate far from the tube centerline, well into the bowed sections of the tube. System 7 drops exhibit breakup into small drops while still in the test section, and further breakup oceurs in the relaxation section, resulting in the formation of many small droplets. The degree of deformation and breakup of eccentrically located drops increases with both $\lambda$ and $V$.

The pressure signal obtained for the large- $\Gamma$ cases differs from the characteristic output shown for the small- $\Gamma$ drops. The most obvious change is that, when the drop deforms into a strand, the pressure signal is no longer periodic and $\delta\left(\Delta P^{+}\right) \rightarrow 0$. Figure 10 indicates that the value of $\Delta P^{+}$is determined mainly by the value of $\sigma$ through the simple replacement of suspending fluid by drop fluid. The details of the time-dependent drop shape are apparently unimportant. This is made evident by noting that a steady pressure reading is obtained for large drops even when a steady drop shape is not attained during the course of an experiment. 

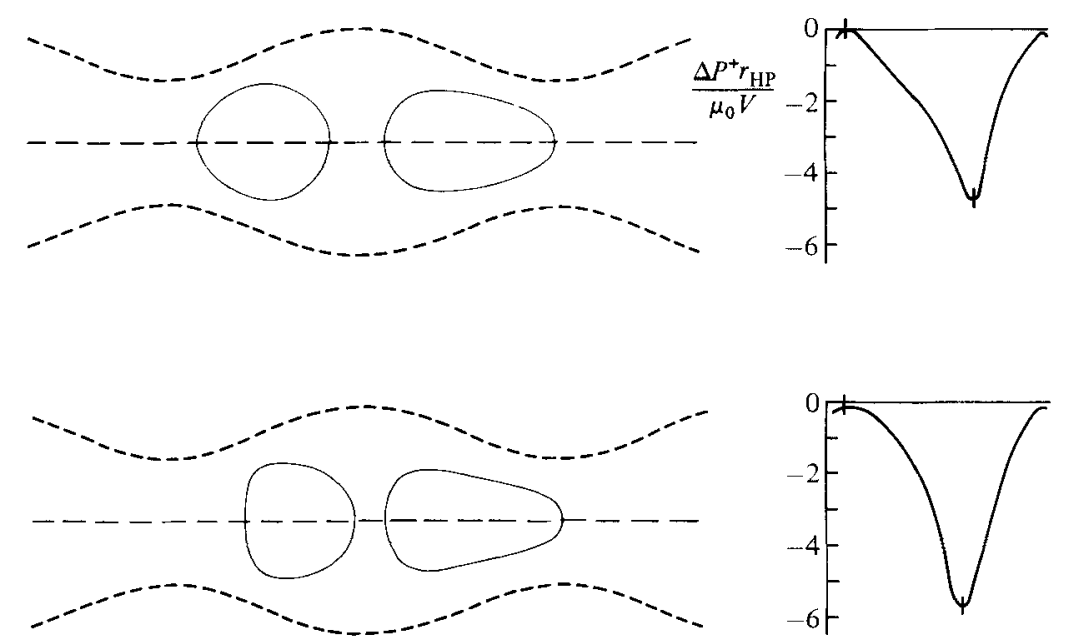

Figure 11. Typical drop shapes and the corresponding dimensionless additional pressure difference for system 1 drops $(\sigma=0.0014$, Newtonian suspending fluid) with $\lambda=0.76, \Gamma=0.50$ (top) and $\Gamma=0.94$ (bottom).

The value of $U / V$ does not depend on the viscosity ratio for these systems, and the data for systems 4-6 are represented by a single curve for each flow rate in figure 7 . The value of $U / V$ for these cases is approximately 1.6, and insensitive to changes in the drop size. The relatively large values for $U / V$, compared with the small- $\Gamma$ cases, simply reflects the fact that most of the drop fluid resides in the central region of the tube. When non-neutrally buoyant drops are used, the drop mobility decreases, but precise values of $U / V$ were not obtained due to drop breakup in the test section.

Relative to the case of large $\Gamma$ and $\sigma=O(1)$ which has been considered in the preceding portions of this section, the deformation and breakup processes are very different for system 1 , where $\sigma=0.0014$. In particular, system 1 drops exhibit breakup without the extreme elongation which preceded breakup for the more viscous drops, though breakup once again occurs only when the values of $\lambda$ and $\Gamma$ are sufficiently large. For the smaller flow rate, for example, breakup occurs when $\lambda>0.8$. It is difficult to observe from the videorecordings the details of the breakup process, but it can be seen that one satellite drop is formed for each oscillation of the wavy-wall tube, until the original drop becomes smaller than the critical size and no further breakup occurs. When the original drop is sufficiently large, the breakup process continues until the drop exits the test section. The first satellite drop, which is always formed in the first oscillation of the test section, is very much larger than the subsequent drops. However, breakup in the first constriction differs from subsequent breakup insofar as the initial shape of the drop for the first constriction is the equilibrium shape attained in Poiseuille flow. This suggests that, in contrast with the case $\sigma \approx 1$ where the drop undergoes gross elongation before breakup, the breakup process for low-viscosity drops depends on the 'initial' shape of the drop when it enters a constriction of the wavy-wall tube. The size of a representative satellite drop is larger if the average flow velocity $V$ and/or the size of the parent drop is increased.

Typical drop shapes and the corresponding pressure signals for cases where no breakup occurs are shown in figure 11. The pressure signals for these cases are periodic, but the effect of the small viscosity of the drop is manifested by negative $\Delta P^{+}$values (ef. figure 6 ). 
It is interesting to note that for the small flow rate, i.e. $\Gamma=0.50$, the maximum value of $\Delta P^{+}$, which is approximately zero, is insensitive to the size $\lambda$ of the drop. At first sight, this result appears contradictory since the overall drop shape necessarily depends on $\lambda$. However, photographs show that the drop shape in the vicinity of the tube throat is identical for drops of all sizes; specifically, the distance between the drop and the tube wall in this region does not change with $\lambda$ for $\Gamma=0.50$. This, apparently, is the explanation for the observed insensitivity of the maximum in $\Delta P^{+}$to variation in $\lambda$. On the other hand, the minimum in $\Delta P^{+}$for these cases is strongly dependent on the size of the drop, which suggests that it is determined substantially by the total volume of drop fluid via the fluid replacement mechanism. For the larger flow rate, i.e. $\Gamma=0.94$, the maximum pressure difference decreases slightly with $\lambda$, but it is still much less sensitive to $\lambda$ than the minimum pressure difference.

enough to undergo breakup, the value of $U / V$ is insensitive to further increases in the drop size $\lambda$. However, the value of $U / V$ for a given drop size increases dramatically with the bulk velocity $V$. This behaviour, too, can be correlated qualitatively with the effects of $\lambda$ and $\Gamma$ on drop shape. We find that the distance between the drop surface and the tube wall in the narrow sections of the wavy-wall tube clearly decreases when $\Gamma$ is made smaller. This apparently causes a greater resistance to drop motion through the throat, which is reflected in the smaller value of $U / V$.

\subsubsection{Comparison with breakup in homogeneous flows}

Since extensive data and some theoretical treatments exist for breakup in two-dimensional homogeneous flows, it is of interest to determine how closely the present results correspond to those results. The data of Grace $(1971,1982)$ for hyperbolic flow show that breakup occurs at a critical value of the dimensionless extension rate $G \mu_{0} b / \gamma$ (where $b$ is the undeformed drop radius), which is a function of the viscosity ratio. The extensional rate $G$ is known exactly for hyperbolic flow, but can only be estimated for wavy-wall tube flow. We use

$$
G=\frac{Q}{\pi L} \frac{R_{0} a}{\left(R_{0}+a\right)^{2}\left(R_{0}-a\right)^{2}}
$$

which is based upon the change in the average velocity between points of maximum and minimum cross-section. Calculated in this fashion, the ranges of values of $G \mu_{0} b / \gamma$ for our experiment are $0.3<G \mu_{0} b / \gamma<0.9$ for $V=0.24 \mathrm{~cm} / \mathrm{s}$, and $0.5<G \mu_{0} b / \gamma<0.9$ for $V=0.46 \mathrm{~cm} / \mathrm{s}$. From our observations, breakup for system 1 drops $(\sigma=0.0014)$ is estimated to occur when the dimensionless extension rate exceeds 0.4. This value compares with a measured critical value of 0.5 for hyperbolic flow. Although it must be admitted that a strict comparison between steady hyperbolic extension and flow through the wavy-wall tube neglects both the rotational component of motion and the wall interactions for the latter case, this result shows that the dimensionless extension rate is at least roughly sufficient to cause breakup of the drop, when interpreted in terms of Grace's criterion. It is important to note, however, that some features of the deformation and breakup process in the wavy-wall tube are quite different from the hyperbolic-flow case. Drop breakup for system 1 drops tends to occur at values of the draw ratio (dimensionless axial length at breakup) which are approximately half those observed in the four-roller device. Also, breakup occurs in a time which is of the order of half the time required in hyperbolic flow. A possible explanation for these differences is that the drop is 
already appreciably deformed in the straight-wall entry section before entering the first constriction of the wavy-wall test section. However, photographs of the drop shape additionally suggest a fundamental difference between the breakup processes for the two flows. In the wavy-wall tube, the shape of the drop is clearly asymmetric in the streamwise direction near the point of breakup, whereas the drop exhibits complete fore-aft symmetry at the point of breakup in the four-roll mill. This suggests that the breakup process depends on the spatial inhomogeneity of the flow rather than simply the existence of a critical local rate of extension. Effects of unsteadiness in the velocity gradient are known to influence breakup in homogeneous flows, and it is possible that the Lagrangian unsteadiness of the flow also plays an important role in determining the conditions for breakup in the wavy-wall tube.

For the cases where $\sigma \approx 1$, the breakup process bears a closer resemblance to breakup in the four-roll mill, at least in the sense that breakup occurs during stress relaxation. We can compare the critical draw ratio for breakup in the two flows. From the data of Grace for viscosity ratios comparable to systems 4,5 and 6 , the critical draw ratios are 5, 9 and 20 respectively. The minimum draw ratios observed for breakup for these systems in the wavy-wall tube are 3,6 and 10 respectively. The values obtained show that breakup definitely occurs at smaller values of the draw ratio in wavy-wall tube flow than in steady hyperbolic flow. The difference again may be a consequence of disturbances in drop shape induced by the tube prior to breakup. The extended drop enters the relaxation section with 'finite-amplitude' wave-like disturbances in shape already present, and these disturbances simply grow during stress relaxation. On the other hand, deformed drops in hyperbolic extension show no capillary waves until the flow is stopped.

It was also noted in $\$ 4.1 .2$ that non-neutrally buoyant drops exhibit a greater degree of elongation than concentric ones for fixed values of the other parameters. Indeed, in many cases, breakup of eccentrically positioned drops occurs in the test section, in marked contrast to neutrally buoyant drops which break up only in the straight-wall relaxation tube for $\sigma=O(1)$. A definition of flow strength based on the ability of the flow to deform a suspended drop (e.g. Olbricht, Rallison \& Leal 1982) would imply that a purely extensional flow, which exists only along the tube centreline, is most efficient at producing drop deformation. It may thus appear surprising, at first, that eccentrically positioned drops exhibit a greater degree of deformation than neutrally buoyant ones, all other factors being equal. However, the flow field in the convergent/divergent tube geometry is not homogeneous, and the magnitude of the velocity gradient, defined as $\left[\nabla v:(\nabla v)^{\mathrm{T}}\right]^{\frac{1}{2}}$, increases monotonically with distance from the tube centreline. Thus it is possible that the flow strength may be greatest away from the tube centreline, even though fluid elements off the centreline experience a rotational component to the flow.

In order to test this possibility, we have examined the detailed kinematics of the undisturbed flow (i.e. no drops present) of a Newtonian fluid through a wavy-wall tube from the solution of the creeping-flow equations of Fedkiw \& Newman (1977). The radial variation in flow strength was calculated at a cross-section (radius $0.35 \mathrm{~cm}$ ) which is midway between maximum and minimum tube diameters. The estimated flow strength first decreases as the distance from the tube centreline increases, but eventually attains a minimum and then monotonically increases as the wall is approached. Indeed, for this particular cross-section, the flow strength at distances from the centreline greater than half the tube radius actually exceeds the flow strength along the tube centreline. Although this result may qualitatively differ for a tube with a greater amplitude of oscillation, the larger flow strength near the tube 

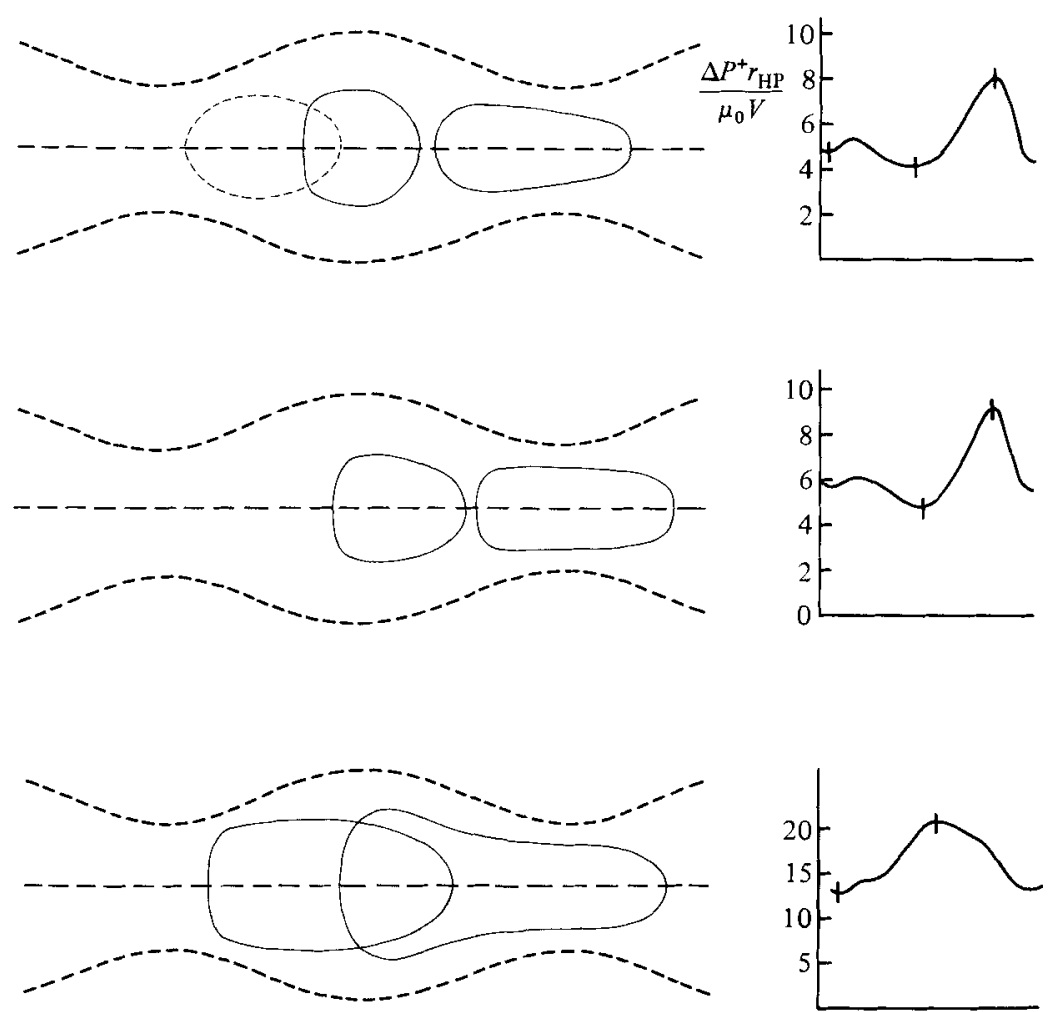

Figure 12. Typical drop shapes and the corresponding dimensionless additional pressure difference for system 11 drops $(0.5 \%$ Separan suspending fluid) with $\lambda=0.76, \Gamma=0.073$ (top); $\lambda=0.76$, $\Gamma=0.097$ (middle); and $\lambda=1.15, \Gamma=0.073$ (bottom).

wall in the present study is consistent with the increased deformation observed for eccentrically positioned drops, relative to neutrally buoyant drops under otherwise similar conditions. It should be noted, however, that another mechanism, namely the hydrodynamic interaction between the drop and the tube wall, can also contribute to increased deformation of eccentrically positioned drops.

\subsection{Viscoelastic fluid systems}

Two concentrations of Separan AP-30 were used as the viscoelastic suspending fluid. The data are presented in figures 12-16.

As in the Newtonian case, the gross shape of the drop is largely determined by the value of the capillary number. Therefore we divide the fluid systems into two classes: small- $\Gamma$ for $\Gamma<0.09$ and large- $\Gamma$ for $\Gamma>0.25$.

\subsubsection{Small- $\Gamma$}

A typical pressure signature and corresponding drop shapes for a system 11 drop are shown in figure 12. For $\Gamma<0.09$ drop deformation differs qualitatively from the shape observed for a Newtonian suspending fluid. The small- $\Gamma$ drop in $0.5 \%$ Separan resembles a prolate spheroid as it passes through the narrow constrictions. The maximum pressure drop is attained when the drop is approximately halfway through the throat of the constriction. Small- $\Gamma$ drops in a Newtonian suspending fluid closely follow the contours of the tube wall, but corresponding drops in $0.5 \%$ Separan respond to the tube geometry more slowly, and they remain tapered even after the 
leading edge of the drop has passed through the eross-section of minimum diameter. Furthermore, the gap between the drop surface and the tube wall, especially in the narrow parts of the tube, is considerably larger than that for the Newtonian systems.

Apart from qualitative observations of the drop shape in the different suspending fluids, another important difference is the relative insensitivity of drop shape, pressure signal and drop mobility to non-viscoelastic parameters of the system such as $\sigma$ and $\Delta \rho / \rho_{0}$. For example, the effect of non-neutral buoyancy on the lateral position of the drop is barely visible, especially in the narrow regions of the tube, and non-neutrally buoyant drops show little asymmetry in shape. Furthermore, the shape of the drop is less sensitive to the value of $\sigma$ than it was for the corresponding Newtonian fluid systems. Evidently, the dominant factor in determining the dynamic properties of the flow is the viscoelastic properties of the suspending fluid, with the other factors that were important for Newtonian fluids playing a secondary role. A similar insensitivity of $\Delta P^{+}$to viscosity ratio was also found in our earlier study of viscoelastic suspending fluids in straight-wall tubes for intermediate-size drops. In that case, it was also speculated that the influence of $\sigma$ on $\Delta P^{+}$(clearly evident for Newtonian suspending fluids) was suppressed by the time-dependent response of the suspending fluid. The Deborah number, measuring the timescale of the flow to the intrinsic relaxation time of the fluid, was maximum in that case for intermediate size drops, since it depended on the relative velocity $U-V$ between the drop and the surrounding fluid. Here we must consider the timescale for droplet deformation relative to the relaxation time of the fluid. This ratio is approximately $D e / \Gamma$, except for the case of very viscous drops (system 11) where the proper ratio is equivalent to the product of the fluid time and the wall shear rate. In any event, for the small- $\Gamma$ cases covered in our experiment, this ratio of timescales is roughly $O(10)$, indicating that the response time for the suspending fluid is expected to dominate the time scale for drop deformation. Thus it is not surprising to find that the influence of drop fluid viscosity on the periodic part of the additional pressure drop is suppressed and that the drop shape responds slowly to the tube geometry under these conditions. For the same reason, the value of the periodic part of the pressure signal $\delta\left(\Delta P^{+}\right)$for the same fluid systems is nearly independent of $\sigma$, at least compared with a relatively strong dependence for Newtonian systems (ef. system $2 b$ and system 3 in figure 6). The average value of $\Delta P^{+}$is, presumably, more closely related to the pressure drop measured in a straight-wall tube. It can be seen from figures 13 and 14 that $\Delta P^{+}$, for fixed values of $\lambda$ and $\Gamma$, is moderately dependent on the viscosity ratio $\sigma$, presumably through simple replacement of fluid, though it is less sensitive to $\sigma$ than in the corresponding Newtonian-fluid case. The averaged $\Delta P^{+}$in the present experiment necessarily reflects contributions over an entire period of the tube. Since the drop spends a majority of each period in configurations where the 'local value' of $\lambda$ is either small or large, the instantaneous relative velocity, analogous to $U-V$, which was found to determine the importance of viscoelastic effects on $\Delta P^{+}$in the straight-wall tube, is also small. Therefore the influence of $\sigma$ on the averaged $\Delta P^{+}$ is not entirely suppressed, even though the influence of $\sigma$ on the time-dependent aspects of the flow is largely gone.

It may be noted, from figure 14, that $\Delta P^{+}$increases with an increase of both $\sigma$ and $\Gamma$ (see system 11 data), whereas $\Delta P^{+}$decreased for a 'similar' increase of $\sigma$ and $\Gamma$ in a Newtonian fluid (see system $2 b$ and 3 in figure 6). In the Newtonian case, when $\Gamma$ was increased, the increased drop deformation caused $\Delta P^{+}$to decrease. In the viscoelastic case, on the other hand, drop deformation does not change appreciably with velocity, and the dominant effect for system 11 (figure 14) is apparently the 


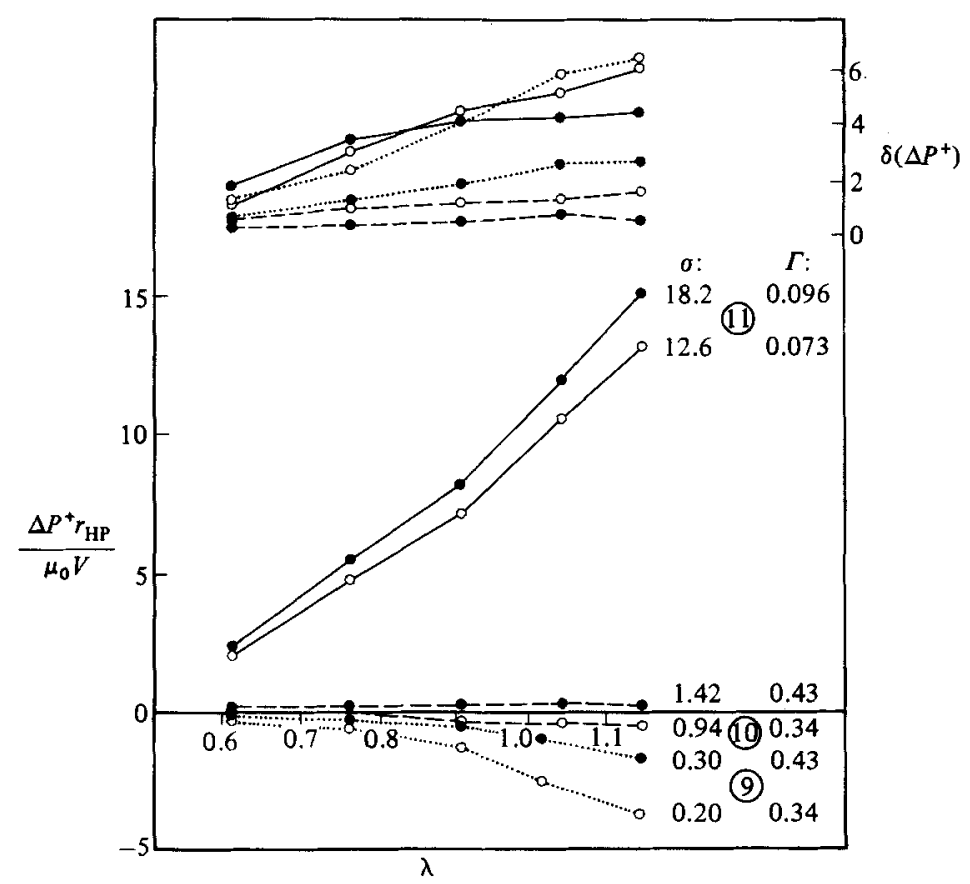

Figure 13. Dimensionless average additional pressure drop and dimensionless magnitude of the oscillatory part as a function of dimensionless drop size for neutrally buoyant systems with $0.5 \%$ Separan.

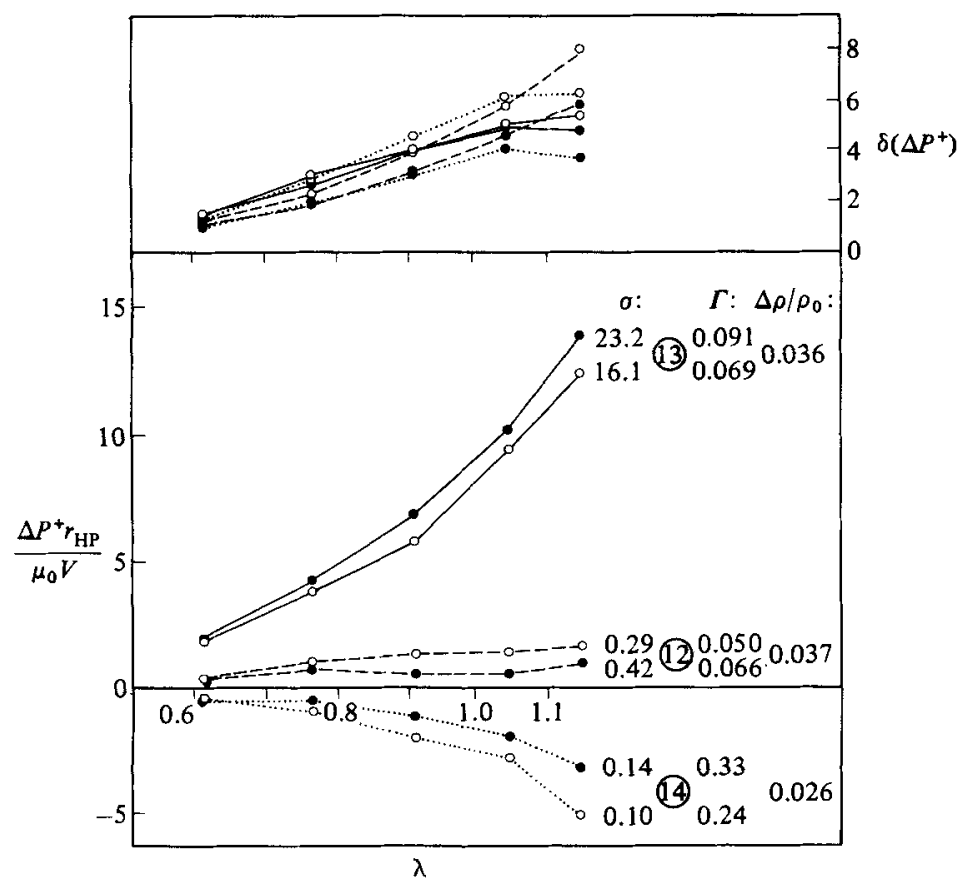

Figure 14. Dimensionless average additional pressure drop and dimensionless magnitude of the oscillatory part as a function of dimensionless drop size for non-neutrally buoyant systems with $0.5 \%$ Separan. 


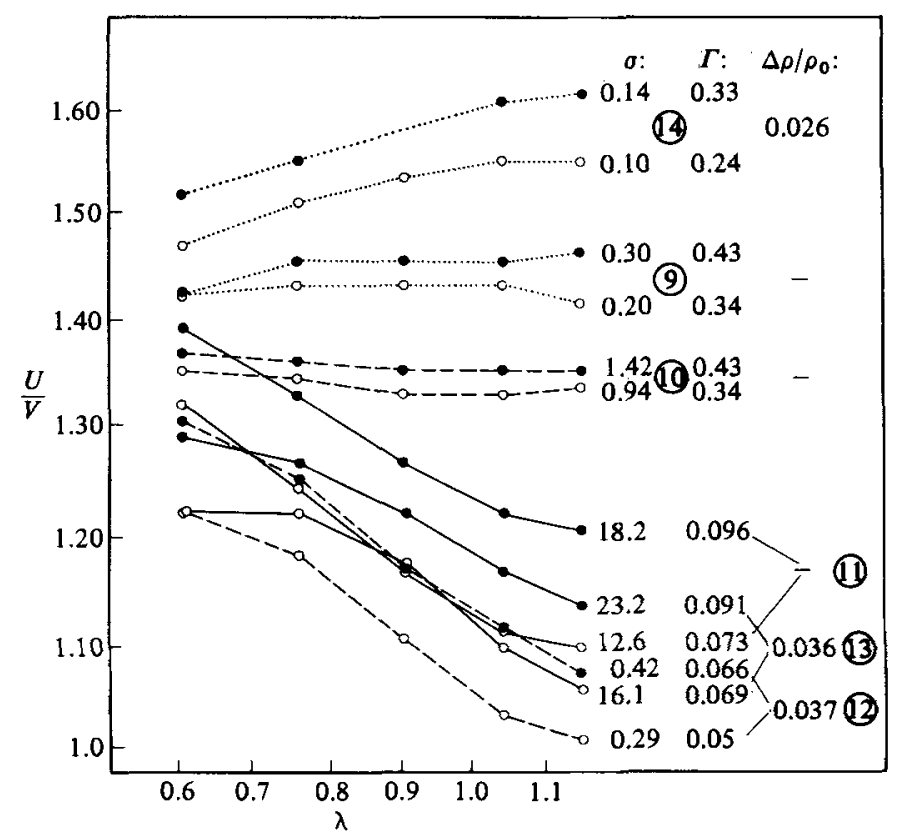

Froure 15. Relative drop velocity as a function of dimensionless drop size for all systems with $0.5 \%$ Separan.

increase in $\sigma$ with $V$ that is a consequence of 'shear thinning' of the suspending fluid. In general, when the suspending fluid is viscoelastic, the value of $\Delta P^{+}$seems to depend mainly on the value of $\sigma$. Detailed results are presented in Olbricht (1980).

The qualitative behaviour for the mobility $U / V$, including the effect of drop eccentricity, closely resembles the Newtonian results for small- $\Gamma$ drops. Indeed, figure 15 indicates that the numerical values are comparable for the various suspending fluids.

\subsubsection{Large- $\Gamma$}

The situation changes when we consider the response of drops for larger values of the capillary number when the suspending fluid is viscoelastic. A typical pressure trace is shown in figure 16 for a system 10 drop. Although there is a greater degree of deformation than in the small- $\Gamma$ case, the shape of the drop remains periodic, and there is no gross elongation of the type observed for the Newtonian systems.

However, another important dispersion process occurs for the $0.5 \%$ Separan suspending fluid which was not observed for any Newtonian system studied. System 9,10 and 14 drops develop tails as illustrated in figure 16. The tail is observed to break up periodically at its end, issuing a satellite drop which trails the parent drop. Like other dispersion processes in this experiment, tail breakup occurs at 'critical ' values of both the average velocity and the drop size. The width of the tail, and therefore the size of the satellites, increases with $V$ and $\lambda$, but decreases slightly as the parent drop continues through the test section. Furthermore, for smaller drops at the small flow rate, the tail forms as the drop passes through each constriction and 'recoils' completely before the drop encounters the next convergent section. As the drop is made larger and/or the flow rate is increased, the tail fails to retract completely in the divergent section before the leading edge of the drop enters the next constriction. When this occurs, the drop develops a 'permanent' tail which grows in 

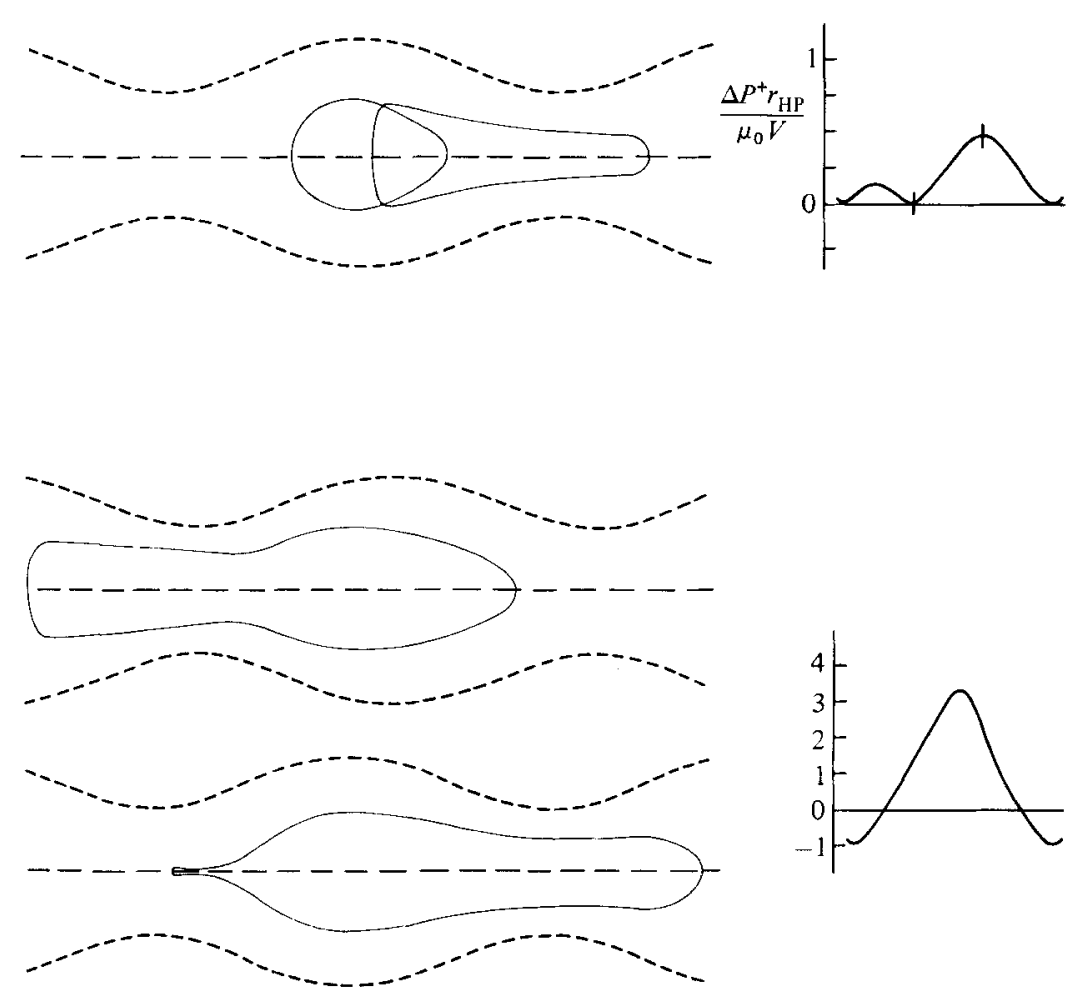

Figure 16. Typical drop shapes and the corresponding dimensionless additional pressure difference for system 10 drops $(\Gamma=0.34,0.5 \%$ Separan suspending fluid) with $\lambda=0.76$ and 1.15 .

length as the drop passes through the test section. In every case, these 'permanent' tails are shed in the relaxation section.

The video recordings show that there are substantial differences in the degree of deformation among the large- $\Gamma$ systems for $0.5 \%$ Separan suspending fluid. Although the shape resembles that observed for the small- $\Gamma$ drops, and the gap widths between the drop and tube wall are comparable, the axial length of the drop is larger in the large- $\Gamma$ cases and increases with $\sigma$. Indeed, system 9 is sufficiently deformed that the leading edge invades the next throat before the tailing edge of the drop has evacuated the preceding one. When this occurs, it has a profound effect on $\delta\left(\Delta P^{+}\right)$, as already discussed. This behaviour is observed for all values of $\lambda$ for systems 9 and 10 (for the large value of $\Gamma$ ) and also for system 14, but only for the largest drop size, which accounts for the curious sign change of the slopes of the $\delta\left(\Delta P^{+}\right)$versus $\lambda$ curves in figure 14. Figure 15 indicates that the effect of $\sigma$ on the deformation of large- $\Gamma$ drops in a viscoelastic suspending fluid is also reflected in the mobility $U / V$ of the drops, which increases with decreasing $\sigma$. Thus the timescale for drop deformation appears to play a significant role for these cases, and, indeed, an estimate of fluid to drop deformation timescales $D e / \Gamma$ is only $O(1)$ for these large- $\Gamma$ systems (compared with $O(10)$ for the small- $\Gamma$ systems considered previously). It is not surprising, then, that non-elastic parameters should influence the drop shape and hence affect the value of $\delta\left(\Delta P^{+}\right)$.

When $1.0 \%$ Separan was used as the suspending fluid, only large- $\Gamma$ systems could be obtained, owing to the relatively large nominal viscosity of the fluid. For the same reason, the response time of the manometer system was comparable to the convective 
time of the periodic flow, and therefore accurate measurements of $\delta\left(\Delta P^{+}\right)$were impossible. The important point is that $\Gamma$ exceeds the values which produced gross elongation of the drops for the Newtonian suspending fluid. Nevertheless, no permanent elongation is noted for the viscoelastic case. However, there are significant qualitative differences in dispersion for the $1.0 \%$ viscoelastic medium.

The drops show increased deformation over the $0.5 \%$ solution, although this is probably a consequence of the larger viscosity of the more concentrated polymer. More importantly, the formation and breakup of the tail for system 15 and 16 drops exhibit different characteristics than those noted for the $0.5 \%$ Separan solution. In particular, breakup of the tail is drastically inhibited in the more concentrated polymer solution. The tail is capable of surviving several (three to ten) oscillations intact before issuing a satellite drop from the tip. The thickness of the tail varies over its length by more than a factor of three without breakup, resulting in a varicose structure which remains attached to the main drop. As a result, fewer satellite drops are issued, usually four or five, compared to eighteen (one per oscillation) in $0.5 \%$ Separan. The increased stability of the tail is in qualitative agreement with the prediction of Chin \& Han (1980) for breakup of a Newtonian jet in a viscoelastic continuous phase. The satellites which are issued are larger and, again, the remaining portion of the tail is shed in the relaxation section of the tube.

The behaviour of $\Delta P^{+}$and $U / V$ with respect to the various parameters is similar to that already reported for the $0.5 \%$ case. A detailed discussion of the results can be found in Olbricht (1980).

\section{Conclusions}

The present experiment shows that the effects of tube geometry on the motion of deformable drops can be substantial and may bear on critical evaluations of tube flow as a prototype problem for multiphase flow through porous media. However, the way in which geometry influences the dynamics of droplet motion depends strongly on the value of the capillary number for the particular situation.

When the suspending fluid is Newtonian, and the value of the capillary number is small, the effects of various parameters on the additional pressure drop and the relative drop mobility measured in wavy-wall tube flow are qualitatively the same as in the case of the straight-wall tube. These measurements are, in a sense, average values, because they reflect local contributions of the various mechanisms which determine flow resistance averaged over a typical period of the wavy-wall tube. But, when the full time-dependent pressure signature is examined, a fundamental effect of the tube geometry is exposed. A major consequence of the wavy-wall tube geometry chosen for this experiment is that peak resistances occur, and they can be very large compared with the corresponding resistance for flow through the straightwall capillary. The maximum pressure drop is always associated with the motion of the droplet through the narrow constrictions of the tube. The appearance of these large peak values suggests that under conditions of a constant imposed pressure gradient, which would describe the appropriate local circumstances for flow through an actual porous structure, a drop could actually be retained at certain locations. In the asymptotic limit of vanishingly small capillary number, these equilibrium drop shapes can be determined; thus our results can be regarded as showing the effects of small, but non-zero, capillary number. It is important to note that the magnitude of the peak resistance for a given situation cannot be predicted from data for flow through a straight-wall tube.

However, when the capillary number is made sufficiently large, the effect of the 
wavy-wall tube geometry is very different. Our experiments show that, for large capillary number, the wavy-wall configuration produces gross elongation of a drop, with subsequent breakup. For the equivalent set of material parameters, no such elongation and breakup is observed in the straight-wall tube. Thus the choice of an appropriate model geometry could affect predictions of the drop size distribution on the macroscale of an actual porous structure.

The drop shape is crucially important to the instantaneous value of the additional pressure drop for small values of the capillary number; not surprisingly, then, the effects of parameters such as viscosity ratio and capillary number are correlated with observed effects on the drop shape. In addition, for small drops, the pressure signature contains many fine details, indicating a complex relationship between drop shape and local tube geometry. For larger drops, these details vanish, and the signals are nearly sinusoidal. However, when the capillary number is large and gross elongation of the drop occurs, our measurements are insensitive to the detailed shape, and the pressure drop is determined primarily by the viscosity of the drop. Under these conditions, two modes of breakup, distinguished by the value of the viscosity ratio, are observed for wavy-wall tube flow. These bear a striking resemblance to breakup in twodimensional hyperbolic extension, and the required rates of extension for breakup are on the same order of magnitude for the two flows. The dispersion processes are important, because, in a real porous structure, a typical drop may pass through such a large number of constriction/expansion sequences that dispersion mechanisms analogous to the ones observed in this experiment would determine the drop-size distribution, especially in the absence of coalescence with other drops.

As in the straight-wall experiment, when the suspending fluid is a polymer solution, both viscometric and time-dependent rheological effects are manifest. To gauge the importance of time-dependent effects, we find that the convective timescale associated with the Lagrangian unsteadiness of the flow owing to the tube geometry should be compared to the intrinsic relaxation time of the fluid. This cannot be predicted $a$ priori, but must be determined by experiment for the specific choice of tube geometry. If the amplitude of the wall oscillation were made progressively smaller, eventually the timescale associated with the relative velocity of the drop with respect to the suspending fluid would become the relevant one to be compared with the intrinsic fluid time.

Although comparison between the cases of Newtonian and viscoelastic suspending fluid is complicated by ambiguity in the definition of the capillary number, it appears that the effect of the polymer solution is to inhibit gross elongation of the drop. Instead, there is a formation of a tail which at first resembles the tail that is formed by a Newtonian drop translating through an unbounded viscoelastic fluid. In this case, the tube geometry is manifested by periodic issuance of satellite drops from the tail.

\section{REFERENCES}

Acrivos, A. \& Lo, T. S. 1978 Deformation and breakup of a single drop in an extensional flow. J. Fluid Mech. 86, 461.

Chin, H. B. \& HaN, C. D. 1979 Studies on droplet deformation and breakup. I. Droplet deformation in extensional flow. J. Rheol. 23, 557.

Chin, H. B. \& HaN, C. D. 1980 Studies on droplet deformation and breakup. II. Breakup of a droplet in non-uniform shear flow. J. Rheol. 24, 39.

Detber, J. A. \& SChowalter, W. R. 1979 Flow through tubes with sinusoidal axial variations in diameter. AIChE J. 25, 638 .

Deiber, J. A. \& Schowalter, W. R. 1981 Modeling the flow of viscoelastic fluids through porous media. AIChE J. 27, 912. 
Elata, C., Burger, J., Michlin, J. \& Takserman, U. 1977 Dilute polymer solutions in elongational flow. Phys. Fluids Suppl. 20 , S49.

Fedkiw, P. \& Newman, J. 1977 Mass transfer at high Peclet numbers for creeping flow in a packed-bed reactor. $A I C h E J .23,255$.

Franzen, P. 1979 Zum Einfluss der Porengeometrie auf den Druckverlast bei der Durchströmung von Porensystemen. I. Versuche an Modellkanälen mit variablem Querschnitt. Rheol. Acta 18, 392.

Grace, H. P. 1971 Dispersion phenomena in high viscosity immiscible fluid systems and application of static mixers as dispersion devices in such systems. Engng Foundation $3 r d$ Res. Conf. on Mixing, Andover, New Hampshire, and Chem. Engng Communs 14 (1982), 225.

Han, C. D. \& Funatsu, K. 1978 An experimental study of droplet deformation and breakup in pressure-driven flow through convergent and uniform channels. $J$. Rheol. 22, 113.

Hinch, E. J. \& Acrivos, A. 1980 Long slender drops in simple shear flow. J. Fluid Mech. 98, 305.

Ho, B. P. \& Leal, L. G. 1975 The creeping motion of liquid drops through a circular tube of comparable diameter. J. Fluid Mech. 71, 361 .

JAMES, D. F. \& McLaREN, D. R. 1975 The laminar flow of dilute polymer solutions through porous media. J. Fluid Mech. 70, 733.

Leal, L. G., Skoog, J. \& Acrivos, A. 1971 On the motion of gas bubbles in a viscoelastic fluid. Can. J. Chem. Engng 29, 569 .

Marshall, R.J. \& Metzener, A. B. 1967 Flow of viscoelastic fluids through porous media. Ind. Engng Chem. Fund. 8, 393.

Michele, H. 1977 Zur Durchflusscharakteristik von Schüttungen bei der Durchströmung mit verdünnten Lösungen aus langkettigen Hochpolymeren. Rheol. Acta 16, 413.

Neira, M. A. \& Payatakes, A. C. 1979 Collocation solution of creeping Newtonian flow through sinusoidal tubes. AIChE J. 25, 725.

Olbricht, W. L. 1980 Ph.D. thesis, California Institute of Technology.

OLBRICHT, W. L. \& LEAL, L. G. 1982 The creeping motion of liquid drops through a circular tube of comparable diameter: the effect of density differences between the fluids. J. Fluid Mech. 115, 187.

OlbRicht, W. L., Rallison, J. M. \& Leal, L. G. 1982 Strong flow criteria based on microstructure deformation. J. Non-Newt. Fluid Mech. 10, 291.

Payatakes, A. C. 1982 Dynamics of oil ganglia during immiscible displacement in water-wet porous media. Ann. Rev. Fluid Mech. 14, 365 .

Payatakes, A. C. \& Neira, M. 1977 Model of the constricted unit cell type for isotropic granular porous media. AIChE J. 23, 922.

Payatakes, A. C., Tien, C. \& Turian, R. M. 1973 A new model for granular porous media. AIChE $J .18,58$.

Payatakes, A. C. \& Tilton, J. N. 1983 Collocation solution of creeping Newtonian flow through sinusoidal tubes: a correction. AIChE J. (to appear).

RALLison, J. M. 1980 Note on the time-dependent deformation of a drop which is almost spherical. J. Fluid Mech. 98, 625.

RaLLison, J. M. 1981 A numerical study of the deformation and burst of a viscous drop in general shear flows. J. Fluid Mech. 109, 465.

Rallison, J. M. \& ACrivos, A. 1978 A numerical study of the deformation and burst of a viscous drop in an extensional flow. J. Fluid Mech. 89, 191.

Saviss, J. G. 1969 Non-Newtonian flow through porous media. Ind. Engng Chem. 81, 18.

SCHowalter, W. R. 1978 Mechanics of Non-Newtonian Fluids. Pergamon.

Sheffield, R. E. \& MEtzNeR, A. B. 1976 Flows of non-linear fluids through porous media. AIChE J. $22,736$.

TAYLOR, G. I. 1934 The formation of emulsions in definable fields of flow. Proc. R. Soc. Lond. A146, 501.

Wasan, D. T., MoNamara, J. J., Shah, S. M., Sampath, K. \& Aderansi, N. 1979 The role of coalescence phenomena and interfacial rheological properties in enhanced oil recovery: an overview. J. Rheol. 23, 181 . 